\title{
Metrics for Evaluating Survivability in Dynamic Multi-Attribute Tradespace Exploration
}

\author{
Matthew G. Richards, ${ }^{*}$ Adam M. Ross, ${ }^{\dagger}$ Nirav B. Shah, ${ }^{\ddagger}$ and Daniel E. Hastings ${ }^{\S}$ \\ Massachusetts Institute of Technology, Cambridge, MA 02139
}

\begin{abstract}
Survivability engineering is critical for minimizing the impact of disturbances to the operation of space systems. To improve the evaluation of survivability during conceptual design, metrics are proposed for the assessment of survivability as a dynamic, continuous, and path-dependent system property. Two of these metrics, time-weighted average utility loss and threshold availability, are then incorporated into a tradespace study on the survivability of future space tug vehicles to orbital debris. A value-based design approach, Dynamic Multi-Attribute Tradespace Exploration, is taken to evaluate survivability based on the relationship between stochastic space tug utility trajectories and changing stakeholder expectations across nominal and disturbed environmental states. Results of the tradespace study show that moderate levels of bumper shielding and access to an on-orbit servicing infrastructure benefit space tugs with large exposed cross-sectional areas while active collision avoidance only delivers value to extremely risk-averse decision makers. Timeweighted average utility loss and threshold availability are found to be discriminating metrics for navigating survivability tradespaces of thousands of design alternatives.
\end{abstract}

\section{Nomenclature}

$A_{T}$

$C S$

$\Delta \mathrm{V}$

$F(t)$

MoME

MTAT

$M T B F$

MTTR

$P_{K}$

$P_{K \mid E}$

$P_{K \mid S S}$

$P_{S}$

$P_{S \mid E}$

$P_{H}$

$P_{K \mid H}$

$R(t)$

$T_{d l}$

$T_{r}$

$\bar{U}_{L}$

$\bar{U}_{t}$

$U(t)$

$V_{e}$

$V(t)$

$V_{x}$

$=$ threshold availability

$=$ campaign-level survivability

$=$ change in velocity

$=$ failure likelihood as a function of time

$=$ measure of mission effectiveness

$=$ mean time above critical value threshold

$=$ mean time between repair

$=$ mean time to repair

$=$ probability of kill

$=$ probability of kill for multiple-shot engagement

$=$ probability of kill from single-shot

$=$ probability of survival

$=$ probability of survival for multiple-shot engagement

$=$ probability of hit (susceptibility)

$=$ probability of kill given hit (vulnerability)

$=$ reliability as a function of time

$=$ time of design life

$=$ permitted recovery time

$=$ time-weighted average utility loss from design utility, $U_{0}$

$=$ time-weighted average utility

$=$ utility delivery over time; multi-attribute utility trajectory

$=$ emergency value threshold

$=$ value delivery over time

$=$ required value threshold

\footnotetext{
${ }^{*}$ Research Assistant, Engineering Systems Division, 41-205, 77 Massachusetts Ave., mgr@mit.edu. Member AIAA.

$\dagger$ Research Scientist, Systems Engineering Advancement Research Initiative. Member AIAA.

${ }^{*}$ Research Assistant, Department of Aeronautics and Astronautics. Member AIAA.

$\S$ Professor of Aeronautics and Astronautics and Engineering Systems, Dean for Undergraduate Education. Fellow AIAA.
} 


\section{Introduction}

S rvivability is the ability of a system to minimize the impact of a finite-duration, environmental disturbance on value delivery. ${ }^{1}$ Given the growth of military and commercial dependency on space systems, ${ }^{2,3}$ identified vulnerabilities in the current U.S. space architecture, ${ }^{4}$ the proliferation of threats, ${ }^{5,6}$ and the weakening of the sanctuary view in military space policy, ${ }^{7,8}$ survivability is an increasingly important consideration during the design of space systems. ${ }^{9}$ Counterintuitively, the risk-averse nature of the space industry, which manifested in the common satellite design elements of redundancy, proven technology, and long design lives, ${ }^{10}$ exacerbates space architecture fragility ${ }^{11}$ by increasing the magnitude of potential downside losses and by reducing the speed at which space capabilities might be reconstituted. Although survivability is an emergent system property that arises from interactions among components and between space systems and their environments, conventional approaches to survivability engineering are often reductionist in nature (i.e., focused only on selected properties of subsystems or modules in isolation). Furthermore, existing survivability engineering methodologies are normally based on specific operating scenarios and presupposed disturbances rather than a general theory with indeterminate threats. As a result, current methods neither accommodate dynamic threat environments nor facilitate stakeholder communication for trading among system lifecycle cost, performance, and survivability. ${ }^{12}$

To address these limitations, a preliminary set of metrics is introduced for the evaluation of satellite survivability in tradespace studies during conceptual design. The metrics are based on a characterization of survivability as the ability of a system to meet required levels of value delivery during nominal and perturbed environmental conditions. To illustrate and test the proposed metrics, the survivability of a low-Earth orbit (LEO) satellite to orbital debris is evaluated for systems incorporating various combinations of susceptibility reduction, vulnerability reduction, and resilience enhancement features. In particular, integrated cost, performance, and survivability trades are performed for an orbital transfer "space tug" vehicle operating in LEO for ten years. Building on previous work, ${ }^{13}$ the impact of bumper shielding, ${ }^{14}$ collision avoidance, ${ }^{15}$ and on-orbit servicing ${ }^{10}$ strategies on space tug encounters with orbital debris is examined.

The modeling approach for testing the survivability metrics consists of four general phases: (1) static MultiAttribute Tradespace Exploration (MATE), ${ }^{16}$ (2) Epoch-Era Analysis, ${ }^{17}$ (3) stochastic simulation of space tug operation, and (4) Monte Carlo analysis. First, a legacy MATE study on space tug vehicles ${ }^{18}$ is extended in which decision maker preferences (i.e., attributes) are quantified and aggregated using multi-attribute utility theory, ${ }^{19}$ and then, candidate satellite designs are assessed in terms of cost and utility using parametric computer models and simulations. Second, Epoch-Era Analysis ${ }^{17}$ is performed in which satellite lifetimes (i.e., Eras) are modeled as sets of discrete time periods with fixed contexts and needs (i.e., Epochs). Applying techniques from network analysis, static tradespaces from MATE are linked over time wherein nodes represent satellite configurations and arcs represent satellite state transitions. Potential system Eras for the candidate space tug designs are constructed by stringing together both baseline (i.e., ambient environment) and disturbance Epochs (i.e., debris conjunction events). Third, a stochastic simulation of space tug operations is performed in which debris impacts are modeled as forced transitions of space tugs in the tradespace to lower utility designs (or end-of-life states). Each run of the simulation produces a utility trajectory (i.e., a plot of multi-attribute utility over time) for a candidate space tug in the tradespace. Fourth, a Monte Carlo analysis is performed across the stochastic, path-dependent utility trajectories, and the survivability metrics are applied.

The paper consists of five sections. Following this introduction, a value-centric definition of survivability is described and illustrated in Section 2. This definition provides a foundation against which the construct validity of existing and proposed survivability metrics may be checked in Section 3. In Section 4, the survivability metrics are applied to a space tug tradespace study. The modeling methodologies and simulation architecture are discussed in detail and several tradespace plots utilizing the metrics are shown. Local response surfaces are also drawn to illustrate the impact of the survivability features in each region of the tradespace. Finally, in Section 5 the implications of the results and priorities for future work are discussed. Development of the dynamic survivability assessment methodology and its associated metrics are illustrative of the challenges and opportunities for better incorporating survivability as a decision metric in tradespaces during conceptual design.

\section{Definition of Survivability}

Survivability may be defined in physical terms as "the capability of a system to avoid or withstand hostile natural and manmade environments without suffering abortive impairment of its ability to accomplish its designated mission." ${ }^{20}$ Survivability may also be defined, more generally, as the ability of a system to minimize the impact of a finite-duration environmental disturbance on value delivery. Value is a subjective measure of benefit from a bundle of consequences that is specified by a stakeholder. ${ }^{21}$ A value-centric conceptualization of survivability is desirable 
during the conceptual design of systems because it provides a fundamental metric for relating system properties to desired stakeholder outcomes. Taking the value-centric perspective during conceptual design empowers decision makers to rigorously evaluate and compare different system concepts in the technical domain (e.g., geosynchronous satellite vis-à-vis low-Earth orbit satellite constellation for a communications mission) using a unifying set of attributes in the value domain (e.g., signal isolation, information rate, information integrity, and data availability). ${ }^{22}$ The ability to consider multiple system concepts using a unifying set of attributes is particularly useful for survivability when original value delivery mechanisms may be blocked by a disturbance.

In addition to conceptualizing survivability as a value-centric property, it is also important to recognize the inherently dynamic nature of survivability. Survivability emerges from the interaction of a system with its environment over time. Depending on stakeholder needs, satellite survivability requirements may allow limited periods during which the system operates in a degraded state, unavailable state, or safe mode. ${ }^{23}$ Recognizing survivability as a dynamic system property informs three general survivability design strategies over the lifecycle of a disturbance: (Type I) susceptibility reduction - the reduction of the likelihood or magnitude of a disturbance, (Type II) vulnerability reduction - the minimization of the disturbance-induced losses on value delivery, and (Type III) resilience enhancement - the maximization of the recovery of value-delivery within a permitted recovery time.

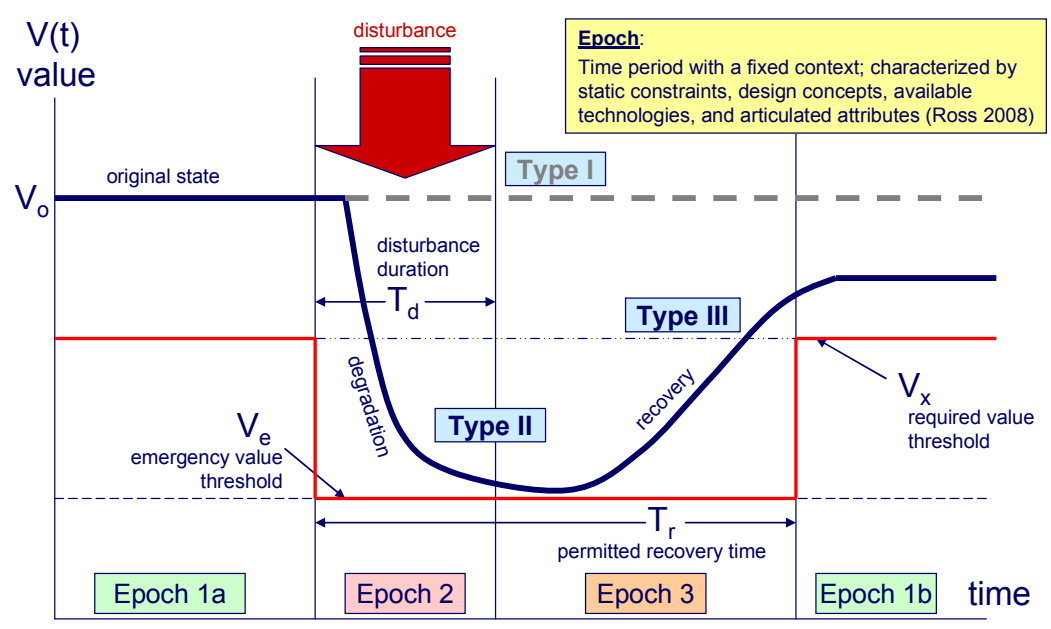

Figure 1. Three Types of Survivability

Figure 1 provides a notional illustration Type I, Type II, and Type III survivability in terms of value delivery over time $[V(t)]$. Time is discretized across four epochs, periods of a fixed context and static stakeholder needs. Following successful value delivery during baseline environmental conditions and stakeholder expectations (Epoch 1a), the system experiences a finite disturbance that degrades performance. Value delivery expectations on the system may be lower during the disturbance (Epoch 2) and in the time period immediately following (Epoch 3) before returning to baseline expectations (Epoch 1b). Type I survivability, depicted as a dashed horizontal line, is achieved if the disturbance fails to reduce $V(t)$ below the required value threshold $\left[V_{x}\right]$ over all of the Epochs. In order to determine whether the system is Type II or Type III survivable, two additional factors must be defined: the minimum acceptable value to be delivered during and immediately after the disturbance $\left[V_{e}\right]$ and the permitted recovery time elapsed past the onset of the disturbance $\left[T_{r}\right]$. In Figure 1, the solid line depicts a system achieving Type II survivability by maintaining $V(t)$ at a level above $V_{e}$ during Epoch 2 and Epoch 3 . The black line also depicts a Type III-survivable system as $V(t)$ recovers to a level above $V_{x}$ within $T_{r}$.

\section{Quantification of Survivability}

\section{A. Existing Survivability Metrics}

Although a diverse array of survivability metrics exist (e.g., range of environments within which an entity remains operational, disturbance threshold above which an entity will cease to function, degree to which performance remains following a disturbance, time required to restore health following a compromising disturbance), ${ }^{1}$ survivability is traditionally incorporated in aerospace design as a binary metric using probabilistic risk assessment. As articulated in Ball's foundational work on combat aircraft survivability, ${ }^{24}$ the probability of 
surviving a one-on-one engagement, $P_{S}$, is the complement of the probability of hit, $P_{H}$ (susceptibility), times the probability of kill given a hit, $P_{K \mid H}$ (vulnerability):

$$
P_{S}=1-P_{K}=1-P_{H} \cdot P_{K / H}
$$

(The susceptibility portion of the calculation, $P_{H}$, can be further decomposed into a five-step event tree or kill chain. For example, at the engagement level, five probability assignments are used to assess system susceptibility: $\mathrm{P}$ [weapon is active], $\mathrm{P}$ [sensor detects], $\mathrm{P}$ [fire control solution is obtained], $\mathrm{P}$ [weapon intercepts], and $\mathrm{P}$ [weapon hits].)

In order to abstract away the complexity associated with dependent shot outcome probabilities, independent shot outcomes are assumed for computing survivability to multiple-shot engagements, $P_{S \mid E} .{ }^{24}$ For example, when the individual shots $P_{K}$ are identical and equal to a single-shot probability of kill $\left(P_{K \mid S S}\right)$ for all $\mathrm{N}$ shots, $P_{S \mid E}$, is:

$$
P_{S \mid E}=1-P_{K \mid E}=\left(1-P_{K \mid S S}\right)^{N}
$$

This logic is extended for computing, campaign-level survivability, $C S$, assuming a campaign of $N$ missions with a constant mission survivability rate:

$$
C S=\left(P_{S}\right)^{N}=\left(1-P_{K}\right)^{N}
$$

While these metrics for assessing single-shot, engagement, and campaign-level survivability provide an elegant mathematical framework for structuring and analyzing the survivability of a system throughout its operational life, challenges remain for the survivability analyst. For example, the reliance on probabilistic risk assessment and its underlying assumptions (e.g., probability distributions are known, disturbance events are independent) is problematic given the complex nature of satellite systems and the known dependencies in a disturbance encounter. For example, the susceptibility of a satellite is not conserved as a constant probability across disturbance encounters if its maneuverability and defensive countermeasures have been reduced by a previous encounter. Another challenge associated with a strictly probabilistic conceptualization of survivability is the binary nature of the metric. The ability of a system to gracefully degrade to reduced levels of capability is frequently cited as a desirable attribute for survivability. ${ }^{1}$ A single $P_{S}$ value applied across systems during conceptual design does not internalize system degradation - a capability which may be useful for trading among design alternatives.

Another challenge for the system analyst relates to the architectural tradespace: how to conduct integrated tradeoffs regarding the varying cost, mission utility, availability, and survivability of alternative system designs? Because these characteristics are interdependent (as survivability design features and operational tactics may lower mission utility and increase cost), pursuing the highest survivability for a given design alternative does not necessarily maximize that system's mission effectiveness. For example, in the lead-up to World War II, 900 pounds of armor were added around the cockpit and fuel tanks of the Brewster F2A "Buffalo" fighter. While these modifications were based on combat data from the European theater, the higher wing loading decreased F2A's service ceiling, maneuverability, and maximum speed - turning a marginally acceptable fighter into an unacceptable one. $^{24}$

To conduct tradeoffs among the availability, survivability, and capability of alternative designs, one approach ${ }^{24}$ is to define a measure of mission effectiveness, $M o M E$, as a product of the three system attributes:

$$
\text { MoME }=\text { Availability } \cdot \text { Survivability } \cdot \text { Capability }
$$

MoME provides a single metric for evaluating the mission effectiveness of systems in an operational environment (and inform strategic decisions such as managed attrition). However, the aggregated metric is of limited prescriptive value during design because the lifecycle costs of alternative designs are not constant. For example, a system with extremely high survivability that can be acquired at greater cost will have a higher MoME but may not allow a useful number of units to be purchased. Conversely, while a lower survivability rate for a satellite within a constellation may be more than offset by a higher availability or capability rate in the MoME computation, the implications of a lower survivability rate may be very negative from a constellation design perspective depending upon the satellite design life and replacement costs.

Other existing quantifications of survivability and related terms include the reliability function and inherent availability. ${ }^{25}$ The reliability function, also known as the survival function, provides the probability that a system will be operational for at least a given time, $t$. 


$$
R(t)=1-F(t)=e^{-t / M T B F}
$$

Inherent availability, $A_{i}$, provides the probability that a system will be operational at any given time in the future, given a mean time between failure, $M T B F$, and mean time to repair, MTTR.

$$
A_{i}=\frac{M T B F}{M T B F+M T T R}
$$

In addition to providing only a binary metric for system health, the reliability function and inherent availability are of questionable construct validity for survivability. Both reliability and availability are concerned with the ability of a system to perform its function under prescribed environmental conditions whereas survivability is concerned with the state of a system that emerges based upon the interaction of that system with external disturbances. Furthermore, the assumed constant failure rates do not apply when disturbances are finite and impulsive in nature.

\section{B. Proposed Survivability Metrics}

While existing survivability approaches have a remarkable legacy for improving the survivability of individual systems (e.g., tremendous combat aircraft survivability improvements from WWII to the present day), evaluation of survivability at higher levels in the system architecture (e.g., constellation, space mission area) is both desirable and required given the increasing interdependencies among systems in networked environments. Based on the discussion in the preceding section, three desirable characteristics for evaluating survivability are: (1) value-based, to allow comparisons across technically-diverse system concepts, (2) dynamic, to allow assessment (and enhancement) of survivability across the lifecycle of a disturbance, and (3) continuous (rather than a discrete, binary characterization), to enable distinction between systems that gracefully degrade and those that fail immediately following a disturbance. In addition, it is also desirable to have survivability metrics that are intuitive as decision metrics and that don't require assumptions to be made regarding the independence of disturbance events. Guided by the definition of survivability in Section 2 and driven by these criteria, this section proposes two metrics for the assessment of survivability in tradespace studies during conceptual design: (1) time-weighted average utility/utility loss and (2) threshold availability.

\section{Time-Weighted Average Utility/Utility Loss}

The development of metrics with construct validity for the survivability definition requires evaluating a system's ability both to minimize utility losses and to meet critical value thresholds before, during, and after environmental disturbances. Given a characterization of a system's value delivery over time, $V(t)$, using a multi-attribute utility function, $U(t)$, the time-weighted average utility loss may be defined:

$$
\bar{U}_{L}=U_{o}-\frac{1}{T_{d l}} \cdot \int U(t) d t
$$

Time-weighted average utility loss may be used to assess the difference between the beginning-of-life, design utility, $U_{o}$, and the time-weighted average utility achieved by a system across operational environments. However, while time-weighted average utility loss is useful for evaluating the impact of various survivability features on a single system, it is less useful for comparisons across systems since $U_{o}$ is not conserved across designs. For example, over-designed systems may have $U_{o} \gg V_{x}$ while $U_{o}$ may approach $V_{x}$ in systems with small performance margins. Therefore, a constant utility loss applied across designs in tradespace will have varying implications in terms of survivability.

To appreciate the survivability implications of a system's ability both to incorporate margin in value delivery and to minimize losses in value, it is necessary to evaluate time-weighted average utility loss relative to $U_{o}$. The difference between these quantities results in time-weighted average utility:

$$
\bar{U}_{t}=U_{o}-\bar{U}_{L}=\frac{1}{T_{d l}} \cdot \int U(t) d t
$$

As the expected (average) temporal utility experienced, time-weighted average utility assesses a system's lifecycle performance over nominal and disturbed environments. However, while this enables continuous 
evaluations to be made across systems regarding ability to minimize utility loss, it is a measure of central tendency that does not internalize ability to meet critical value thresholds.

\section{Threshold Availability}

Threshold availability, $A_{T}$, is proposed as a survivability metric to evaluate the ability of a system to meet critical value thresholds. $A_{T}$ is defined as the ratio of mean time above thresholds to the total design life.

$$
A_{T}=\frac{M T A T}{T_{d l}}
$$

While structured similarly to $A_{i}, A_{T}$ is unique in that the critical value threshold varies across nominal, disturbance, and recovery Epochs. Utility losses, such as degradation or finite outage of capability, may be allowable in disturbance environments (i.e., $V_{x}$ and $V_{e}$ both correspond to the minimally-acceptable level of value delivery, $U\left(x_{i}\right)=0$, during their respective Epochs).

One implication of value thresholds changing as a function of disturbance encounters is that definition and scale of a utility axis will vary across epochs and is therefore not consistent across the system lifecycle. This presents a challenge: how to present lifecycle utility data if the utility axis is a moving target? One reasonable approach is to assume that the attribute set composing the utility function is constant throughout the mission while varying the acceptability range of the single-attribute utility functions during the disturbance and recovery Epochs. In this way, time-weighted average utility is computed from the emergency $U\left(x_{i}\right), V_{e}$ is set as the level of attributes corresponding to zero utility during emergency $U\left(x_{i}\right)$, and $V_{x}$ is set as the level of attributes corresponding to zero utility during nominal $U\left(x_{i}\right)$ (Figure 2). This approach is equivalent to saying that during an emergency, people are willing to accept lower levels of their performance metrics, but will raise those expectations back to nominal levels following the emergency.

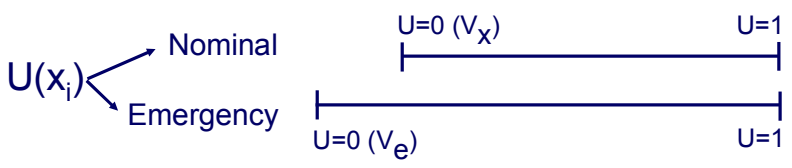

Figure 2. Setting Required and Emergency Value Thresholds

\section{Application of Survivability Metrics}

Having discussed existing and proposed metrics for evaluating survivability during conceptual design, an extended example is now presented to test the survivability metrics in a tradespace study. The system evaluated is a space tug, for which an existing, well-understood tradespace model is available. ${ }^{18}$ The survivability is defined in terms of sustained tug performance in an orbit threatened by debris. Building on previous work that investigated the passive survivability benefits provided by bumper shielding, ${ }^{13}$ the survivability model presented here adds the active survivability strategies of collision avoidance and on-orbit servicing to the tradespace. Following an overview of the modeling methodologies employed, this section summarizes the software architecture of the space tug model and then applies various survivability metrics to a set of experimental tradespaces. The purpose of the section is to both test the amenability of the metrics as decision criteria and explore how each might be incorporated into traditional cost-utility tradespaces.

\section{A. Modeling Methodology: Dynamic Multi-Attribute Tradespace Exploration}

Multi-Attribute Tradespace Exploration (MATE) is a conceptual design methodology that applies decision theory to model and simulation-based design. Decoupling the design from the need through tradespace exploration, MATE is both a solution-generating as well as a decision-making framework. Figure 3 summarizes the MATE process. At a high level, implementing the MATE approach to system design involves three activities. First, the preferences of a decision maker (i.e., architecture evaluation criteria) are defined and specified with attributes (i.e., decision maker-perceived metrics that measure how well decision maker-defined objectives are met). These attributes are aggregated using Multi-Attribute Utility Theory to arrive at an aggregate utility function (a dimensionless metric of user satisfaction ranging from 0 , minimally acceptable, to 1 , highest of expectations). Second, the attributes are inspected, and various design variables (i.e., designer-controlled quantitative parameters 
that reflect aspects of a concept, which taken together as a set uniquely define a system architecture) are proposed. Each possible combination of design variables constitutes a unique design vector, and the set of all possible design vectors constitutes the design-space. Third, a system model is developed to assess the cost and utility of the candidate architectures. In a static-MATE analysis, a limited number of Pareto-efficient designs may then be selected for more rigorous analysis. ${ }^{13}$

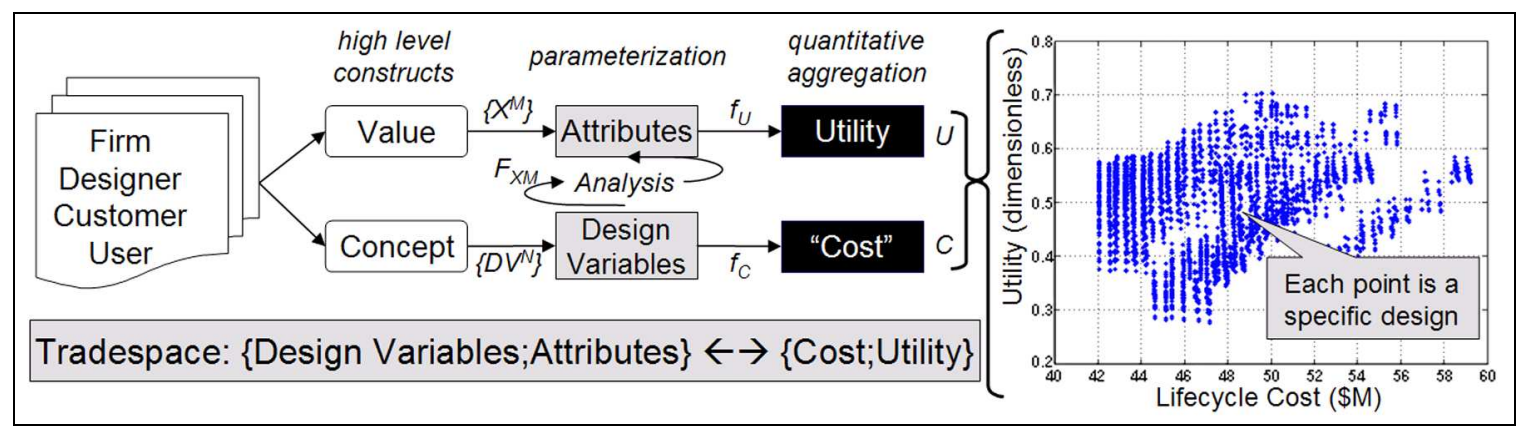

Figure 3. Multi-Attribute Tradespace Exploration (MATE) ${ }^{16}$

In a dynamic MATE study, network analysis is applied to a series of temporally-linked tradespaces, enabling the quantification of various "ilities" (i.e., lifecycle system properties such as survivability) as decision metrics across design alternatives. In order to incorporate time into traditionally static cost-utility tradespaces, Epoch-Era Analysis is employed whereby a system lifecycle or "Era" is modeled as a set of discrete Epochs (Figure 4). Equivalent to short-run analysis in economics, a given Epoch defines a scenario in which constraints, design concepts, available technology, and articulated attributes remain fixed. ${ }^{17}$ Given the focus on survivability in this paper, the only changes across Epoch boundaries in the space tug model are finite-duration disturbance events in the environment (i.e., debris impacts) and changing stakeholder expectations (i.e., short-term relaxation of stakeholder expectations during disturbance and recovery Epochs).

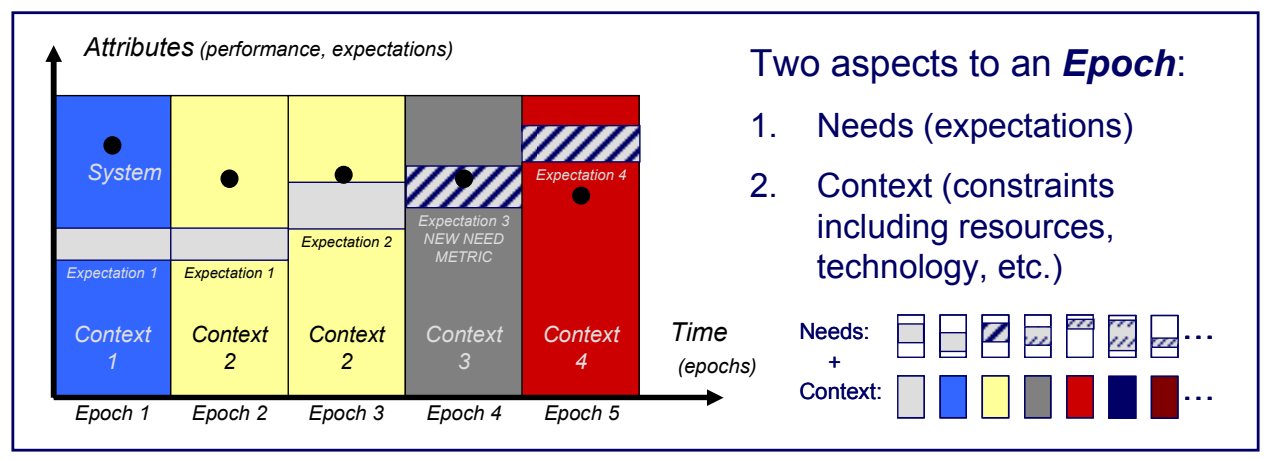

Figure 4. Epoch-Era Analysis ${ }^{17}$

Just as a movie reel approximates motion by stringing together multiple static pictures in quick succession, dynamic MATE strings together multiple static tradespaces over time to approximate dynamic system contexts and expectations. System states may be represented within an Epoch using a network tradespace. In the limit that the design vectors in the tradespace represent all meaningful system states, a dynamic model may be approximated within the tradespace as transitions between design vectors over time. An accessibility matrix specifies the ability of one design to transition to another, and a transitions rules matrix specifies the time and cost incurred for a given transition. When focused on survivability, transitions in a dynamic MATE study primarily comprise (1) forced transitions to lower utility states during disturbance events and (2) transitions to higher utility states during recovery.

\section{B. Design Problem: Space Tug Survivability to Orbital Debris}

A space tug is a vehicle designed to rendezvous and to dock with a space object; to make an assessment of its current position, orientation, and operational status; and either to stabilize the object in its current orbit or to move the object to a new location with subsequent release. ${ }^{26}$ A baseline MATE study explored the tradespace for a 
general-purpose servicing vehicle. ${ }^{18}$ Three attributes formed the multi-attribute utility function: total $\Delta \mathrm{V}$ capability, capability of the grappling system, and response time. To provide these attributes, three design variables were considered in subsequent modeling activities: manipulator mass, propulsion type, and fuel load. A full-factorial, cost-utility tradespace was enumerated - featuring 128 designs - by inputting each possible combination of design variables into (1) a parametric cost estimation model and (2) a physics-based performance model. Building on both the baseline architecture trade study and a previous survivability study that investigated only passive bumper shielding, ${ }^{13}$ the dynamic MATE model presented in this section incorporates passive and active survivability strategies into the tradespace.

\begin{tabular}{|c|c|c|c|c|c|c|}
\hline \multicolumn{7}{|c|}{ Design Variables } \\
\hline & & & & ----- & $=--$ & -----2 \\
\hline Manipulator Mass & \begin{tabular}{|l|} 
Propulsion Type \\
\end{tabular} & \begin{tabular}{|l|} 
Fuel Load $(\mathrm{kg})$ \\
\end{tabular} & I & \begin{tabular}{|l|} 
Shield Mass $(\mathrm{kg})$ \\
\end{tabular} & Servicing & Collision Avoidance \\
\hline \begin{tabular}{|l|} 
Low $(300 \mathrm{~kg})$ \\
\end{tabular} & \begin{tabular}{|l|} 
Storable bi-prop \\
\end{tabular} & \begin{tabular}{|c|}
30 \\
\end{tabular} & I & \begin{tabular}{|c|}
30 \\
\end{tabular} & no & \begin{tabular}{|c|} 
no \\
\end{tabular} \\
\hline Medium $(1000 \mathrm{~kg})$ & Cryogenic bi-prop & 100 & & 100 & yes & yes \\
\hline High (3000 kg) & Electric (NSTAR) & 300 & $\mathbf{I}$ & 300 & & \\
\hline Extreme $(5000 \mathrm{~kg})$ & Nuclear Thermal & 600 & I & 500 & & \\
\hline & & $\begin{array}{c}3000 \\
10000 \\
30000\end{array}$ & & $=\frac{1000}{----}$ & & \\
\hline
\end{tabular}

Figure 5. Space Tug Design Options $(\mathbf{n}=2560)$

While the baseline MATE study focused on trades among the efficiency and response time of the propulsion system, grappling capability, and cost, the addition of survivability features to the design space introduces new trades between cost, design utility (i.e., utility achieved under nominal conditions at beginning-of-life), and survivability. Figure 5 depicts the six design variables used to specify a given design vector (i.e., design alternative), including three variables for survivability features. Each of the three variables is intended to drive a particular type of survivability. For Type I survivability, susceptibility reduction is achieved through active collision avoidance of cataloged debris objects. Susceptibility is also reduced by selecting design vectors of small spacecraft (which will have smaller exposed cross-sectional areas to the debris flux). For Type II survivability, vulnerability is reduced by selecting higher levels of bumper shielding. In addition, vulnerability may also be reduced by selecting space tugs of higher capability - potentially providing margin to degradation losses. For Type III survivability, resilience may be enhanced by paying an upfront insurance fee for on-orbit servicing and repair in the event of noncatastrophic debris impacts.

Figure 6 provides a high-level perspective of how the space tug survivability model is implemented over seven general steps. First, 2560 space tugs designs are defined through a full-factorial sampling of the six enumerated design variables.

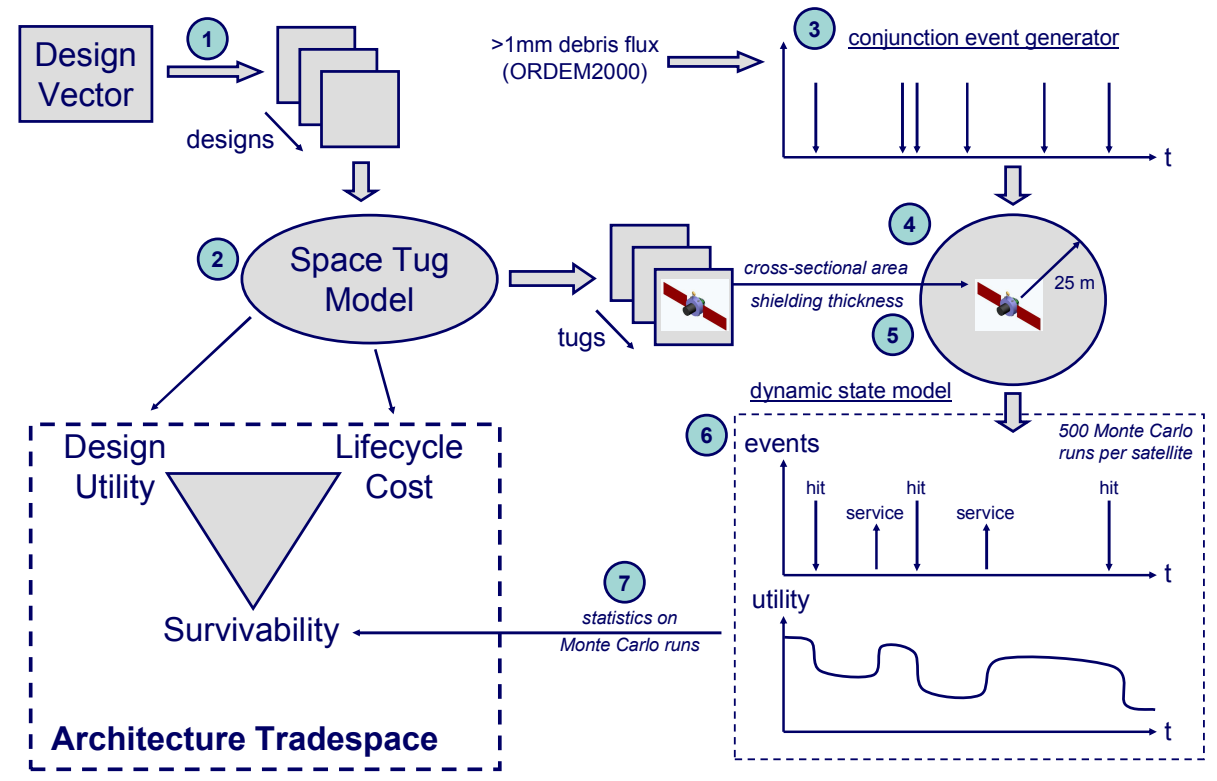

Figure 6. Model Overview 
Second, the legacy space tug model and stakeholder multi-attribute utility function are used to assess the lifecycle cost and design utility of each design vector. After imposing dry mass and cost penalties for the shielding (variable mass penalty as specified by design variable), servicing ( $\$ 100$ million dollar insurance fee and $50 \mathrm{~kg}$ docking ring $^{27}$ ), and warning service for active collision avoidance (assumed fee of $5 \%$ of baseline space tug cost), the legacy model calculates the lifecycle cost and design utility of the expanded design vector.

Third, conjunction events - defined here as the passage of debris object greater than $1 \mathrm{~mm}$ in diameter within 25 meters of the satellite - are generated according to a Poisson process where the arrival rate is determined by the debris flux. Assuming that the space tugs are launched in 2009 into an $800 \mathrm{~km}$ circular orbit at $42.6^{\circ}$ inclination (a relatively populated region of LEO), the orbital debris flux is extracted from NASA's ORDEM2000 model.* Flux is assumed isotropic and constant over the ten-year operational life.

Fourth, given a conjunction event, the baseline susceptibility of each satellite to a debris hit is computed as the ratio of the satellite cross-sectional area to the cross-sectional area of the conjunction sphere. Figure 7 shows a histogram of the probability of a hit given a conjunction event for all candidate space tug designs.

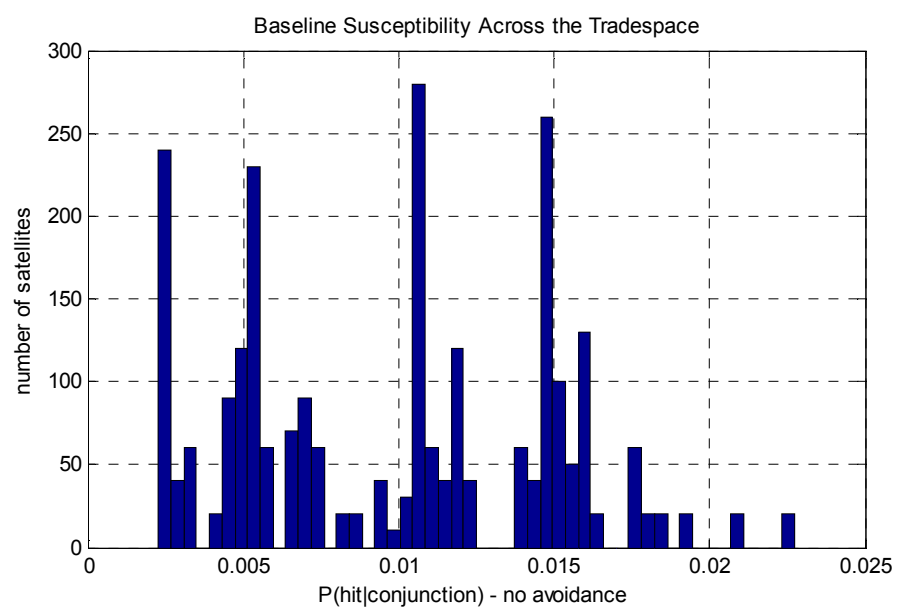

Figure 7. Conjunction Outcomes

Fifth, given a hit, the probability of an encounter with one of three possible sizes of debris is computed: (1) a small debris impact, (2) a medium debris impact, or (3) a large debris impact. (Debris of $<1 \mathrm{~mm}$ diameter are termed micro and are assumed to have no impact.) Table 1 enumerates the impact outcomes as reported in Ref. 28 as well as the modeling assumptions made regarding the degradation of a space tug design for a given size impact.

Table 1. Impact Outcomes

\begin{tabular}{|c|c|c|c|c|}
\hline \multirow{2}{*}{$\begin{array}{c}\text { debris } \\
\text { diameter }\end{array}$} & micro & small & medium & large \\
\hline $\begin{array}{c}\text { impact } \\
\text { outcome }\end{array}$ & degradation & damage & $\begin{array}{c}\text { severe } \\
\text { damage }\end{array}$ & satellite loss \\
\hline $\begin{array}{c}\text { modeling } \\
\text { assumption } \\
(7 \mathrm{~km} / \mathrm{s})\end{array}$ & no impact & $\begin{array}{c}10 \% \text { chance } \\
\text { of loss in } \\
\text { capability } \\
\text { level }\end{array}$ & $\begin{array}{c}\text { loss in } \\
\text { capability } \\
\text { level }\end{array}$ & $\begin{array}{c}\text { end-of-life / } \\
\text { collision } \\
\text { avoidance } \\
\text { with 99\% } \\
\text { success }\end{array}$ \\
\hline
\end{tabular}

While the probability of encounters with micro and large debris objects are constant across space tug designs, the probability of encounters with small and medium debris objects varies relative to the maximum debris size impact that can be sustained by the bumper shielding of a given space tug without deflection, rupture, or spalling. (The size of objects large enough to penetrate the bumper shield varies based upon the thickness of the shield, which in turn depends on the shield mass and satellite body area. Using an empirically-derived bumper shield model, ${ }^{14}$

\footnotetext{
${ }^{* *}$ See http://orbitaldebris.jsc.nasa.gov/model/engrmodel.html.
} 
empirically-derived relationships for relating debris diameter to debris mass, ${ }^{29}$ and assuming the approximate average orbital relative velocity in ORDEM 2000 of $7 \mathrm{~km} / \mathrm{s}$, the maximum debris diameter for a small hit is computed.) Table 1 illustrates this variable threshold as $\mathrm{x} \mathrm{cm}$. Then, given the three critical debris diameter thresholds of $1 \mathrm{~mm}, \mathrm{x} \mathrm{cm}$, and $10 \mathrm{~cm}$ for each satellite, and using the spatial density of debris of those diameters in ORDEM2000 (Figure 8), the cumulative probability that the intercepting particle of debris falls into each of the debris size bins is computed.

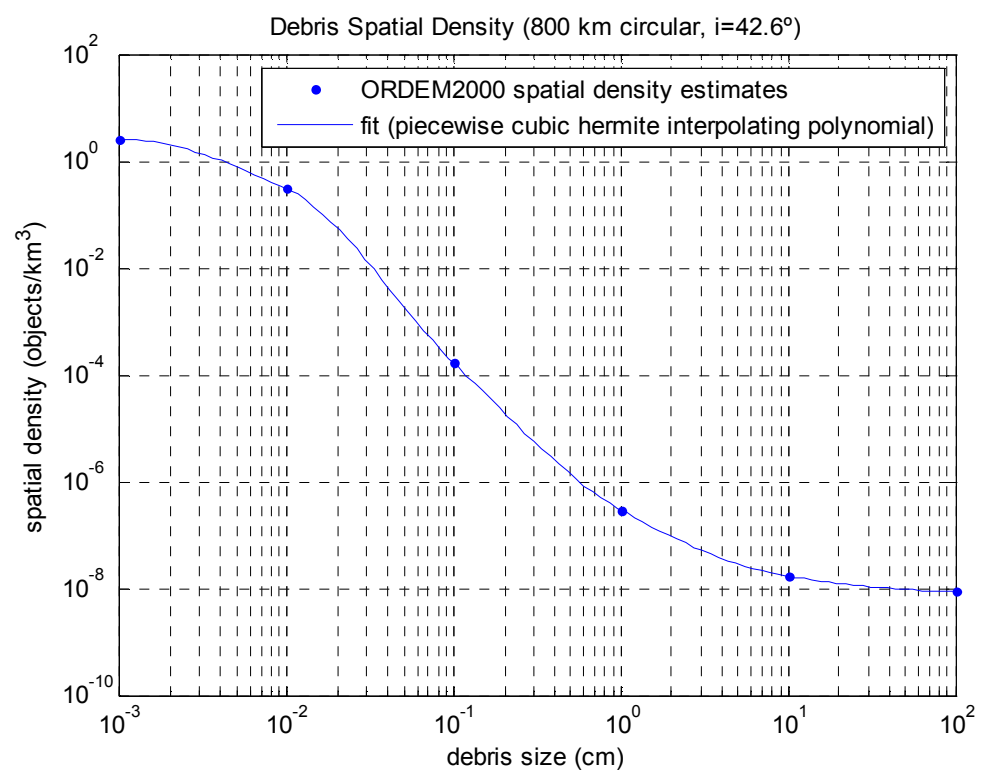

Figure 8. Spatial Density of Debris

Because the outcome of a conjunction event is probabilistic and path-dependent in nature, a Monte Carlo analysis is performed around the sixth step. The 10-year operational life of each space tug is simulated 500 times to generate a diverse range of possible utility trajectories. While $\Delta \mathrm{V}$ expenditures from normal mission operations slowly degrade capability (at a rate of $1 / 15$ of the $\Delta \mathrm{V}$ budget per year), the most significant drivers of utility change are debris impact events and servicing. For each conjunction in a given run of the simulation, the probability of an encounter with a given type of debris is used to probabilistically insert disturbance Epochs of small, medium, and large debris encounters. The implications of a given encounter may be uneventful, non-catastrophic (utility degradation), or catastrophic ( $U=0$ for the remainder of the 10 -year period). In the event of an encounter with a large debris object, space tugs that did not invest in collision avoidance are catastrophically impacted while those tugs investing in avoidance are able to maneuver away from these cataloged objects with $99 \%$ success. ${ }^{15}$ In the event of a medium encounter, the debris object is too small to track (and hence avoid) but too large to completely shield. Space tug degradation from this penetrating impact is modeled as a forced transition in the tradespace network whereby the grappling capability design variable of the space tug is reduced by one discrete level. For example, if a space tug with "low" grappling capability $\left(U_{i}=3\right)$ is subjected to a non-catastrophic hit, grappling capability drops to "none" $\left(U_{i}=.0\right)$, a minimalistic operational state in which no utility is derived from grappling. For those design vectors including a servicing option, an on-orbit repair is attempted following a non-catastrophic hit. Successful servicing missions restore grappling capability to the original (baseline) level in the design vector. (A given servicing mission is assumed to have a $70 \%$ success rate. Response times are lognormally distributed with a mean of six months and a standard deviation of a year.) In the event of an encounter with a small debris object, any debris hitting the bumper shield is stopped. However, as bumper shielding does not completely cover the satellite (as it is concentrated around the most susceptible and vulnerable regions), a $10 \%$ probability of noncatastrophic impact is assumed.

Having sampled 500 utility trajectories over the distribution of possible impact and recovery sequences for each of the 2560 space tug designs, summary statistics are collected in the seventh and final step of the model to measure central tendency of lifecycle survivability. These statistics include the existing and proposed metrics of survivability discussed in Section 3. 


\section{Results}

This section presents the results of the dynamic MATE analysis. After showing the impact of the survivability features on the baseline tradespace, results from the dynamic state characterization of space tug designs are shown. These results include sample utility trajectories, application of the survivability metrics within the baseline tradespace, and the introduction of a new survivability tradespace that allows integrated trades to be made among cost, performance, and survivability. The section concludes with a response surface analysis of each survivability feature-investigating the impact of shielding, collision avoidance, and servicing on each space tug design.

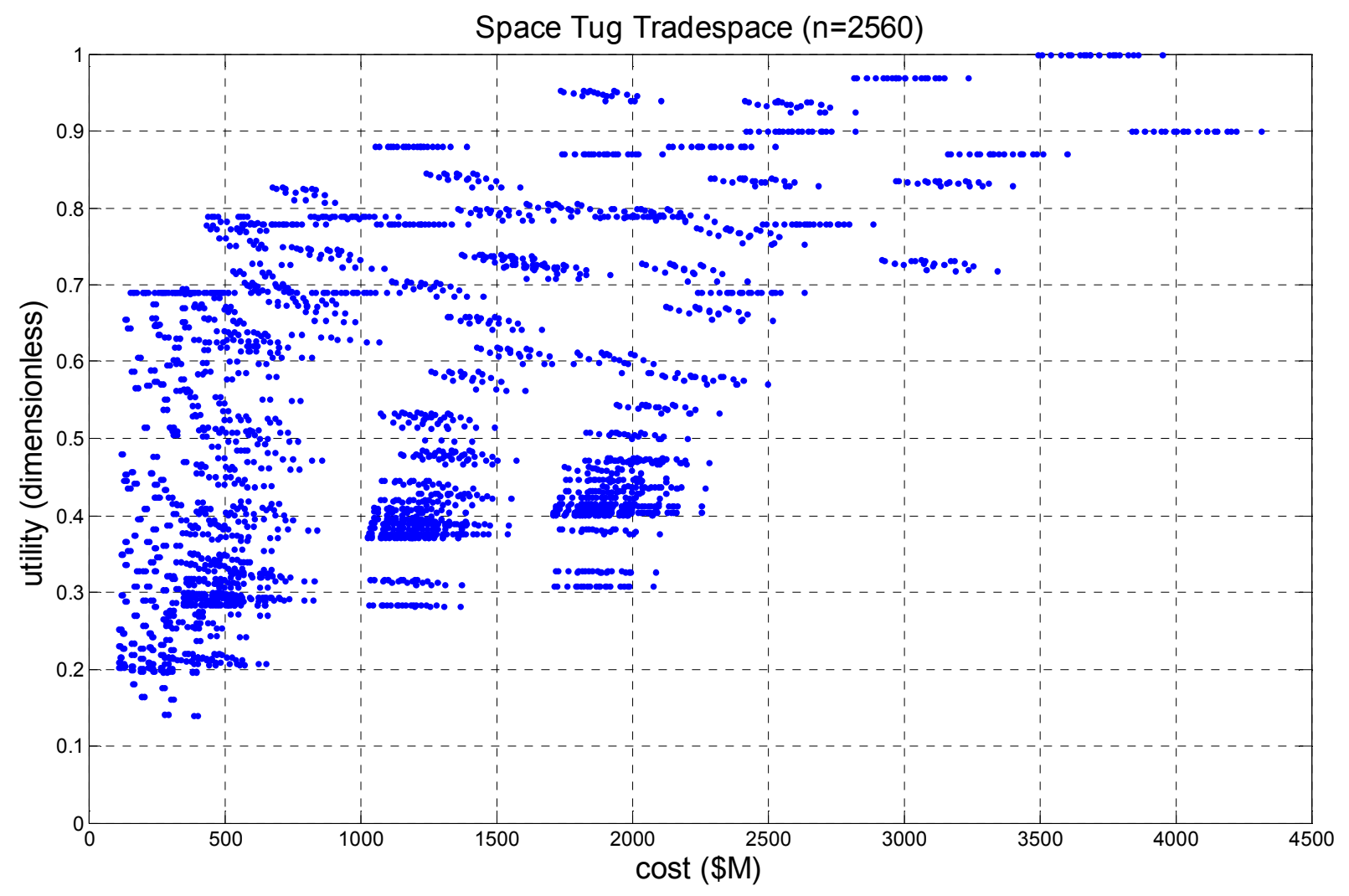

Figure 9. Baseline Tradespace

As discussed in Ref. 18, the original space tug analysis showed an interesting trade-off among cost, grappling capability, and responsiveness. Several different classes of vehicles occupied the Pareto front, including small chemically-fueled vehicles for low $\Delta \mathrm{V}$ maneuver, servicing, and inspection; small electric vehicles that could apply considerable $\Delta \mathrm{V}$, although slowly; and large, expensive vehicles that could apply large $\Delta \mathrm{V}$ 's quickly but only at high launch costs (for chemical fuel) or high development cost and risk (for nuclear-thermal vehicles).

The current analysis adds 20 possible combinations of survivability features for each of the design alternatives in the baseline set of 128 design vectors. Figure 9 depicts the new space tug tradespace of 2560 design alternatives. The horizontal axis is cost (in millions of dollars) and the vertical axis is multi-attribute utility, which is a function of $\Delta \mathrm{V}$ capability, grappling system capability, and response time. Each point is a design vector-a unique combination of the six design variables (Figure 5). The Pareto-efficient region of the tradespace-where utility is highest for a given expenditure-is located in the top-left region in the plot.

During normal operating conditions, space tug value is affected negatively by the addition of survivability features which only add cost and reduce $\Delta \mathrm{V}$ capability. This effect is uneven, with smaller, less expensive vehicles being affected the most. Families of vehicles differentiated only by survivability features are visible as clusters. On the far left of the figure, many clusters run to the right (added costs) and also sharply down (decreased utility), indicating that adding survivability moderately increases cost but strongly degrades utility. Other families of vehicles (e.g., those near the center) show cost increases but without strong impact on utility. Finally, a few families (mostly near the top of the chart) show only cost increases because these electric propulsion vehicles actually have 
excess $\Delta \mathrm{V}$ capacity (i.e., exceeding upper range of $\Delta \mathrm{V}$ utility curve), so the added weight of the shielding and docking interface (for servicing) does not decrease utility.

The baseline cost-utility tradespace is valuable to decision makers during front-end design as it enables costbenefit analysis across thousands of concepts-avoiding the limits of local point solutions-and, most fundamentally, maps the decision maker preference structure onto the design space. However, the tradespace dimensions are of limited applicability to a survivability analysis of a space tug. The survivability design variables are shown to only add cost and/or reduce utility in this representation, meaning that every perturbation off of one of the baseline 128 designs (i.e., full-factorial of first three design variables in Figure 5) is dominated. Given that survivability features may add value to the space tug in the presence of disturbances - and given the problems associated with incorporating "ilities" such as survivability as an attribute within multi-attribute utility functions ${ }^{13}$ additional axes for survivability metrics must be added to the tradespace for a decision maker to have an integrated perspective of cost, performance, and survivability.

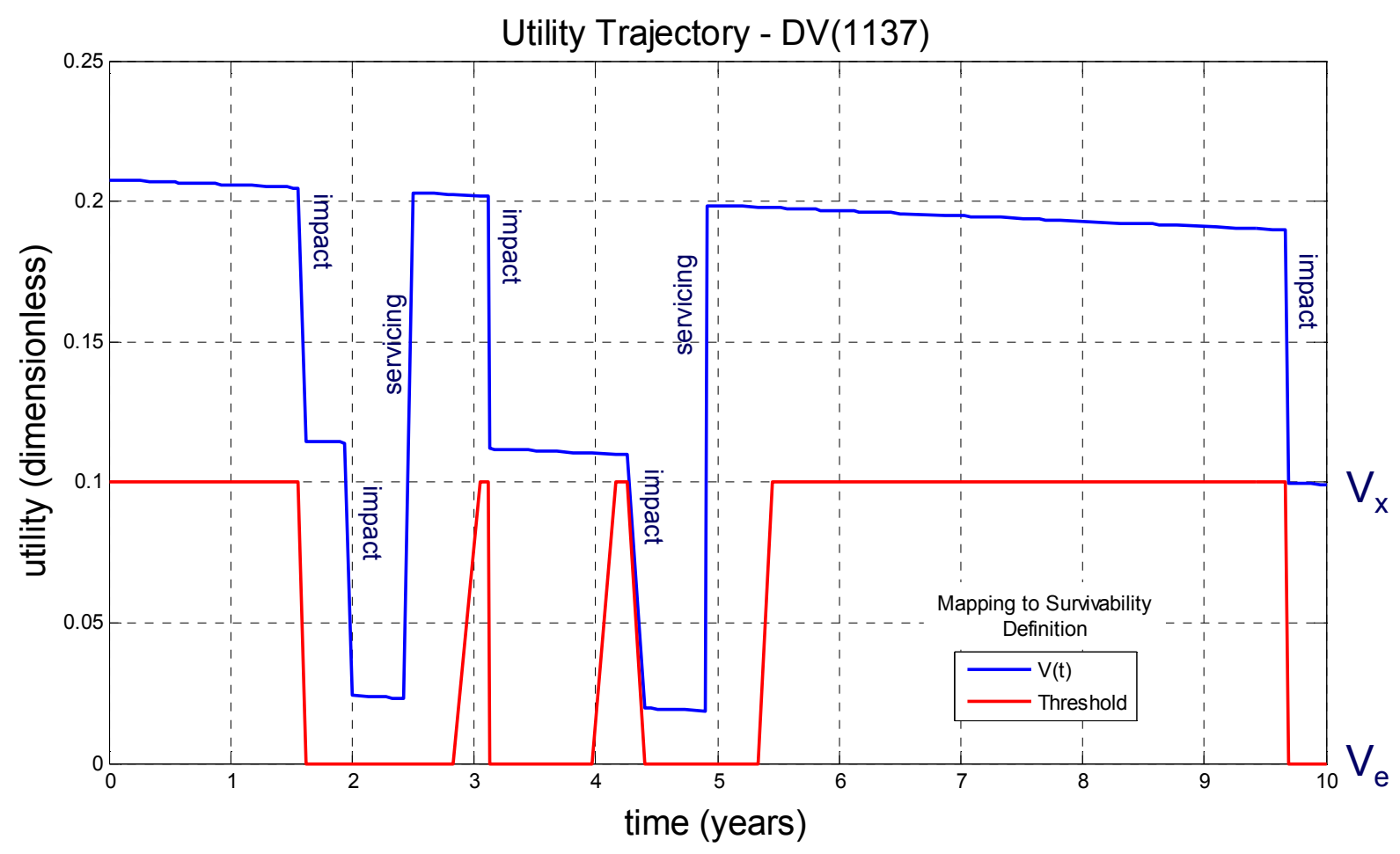

Figure 10. Sample Utility Trajectory

As discussed in Section 2, survivability is an emergent system property which may be defined as the ability of a system to maintain value delivery within stakeholder-defined thresholds over the lifecycle of a disturbance. The dynamic space tug model operationalizes this definition by simulating utility trajectories of alternative designs in the presence of orbital debris events. Figure 10 presents a sample utility trajectory output from the model, illustrating $V(t)$ (i.e., dynamic multi-attribute utility) over a possible 10-year operational life. Following normal degradation during the first 18 months of operation (modeled as nominal $\Delta \mathrm{V}$ expenditures), two non-catastrophic debris impacts occur in quick succession during the latter part of the second year. Due to the reduction in expectations from $V_{x}$ to $V_{e}$ for a year following the first impact (and renewed following the second impact), $V(t)$ does not pass below the value threshold. The first debris impact prompts a request for servicing that is successfully filled during the second year. A similar sequence of events - consecutive debris hits followed by successful servicing - occur between the third and fifth years. In both cases, large quantities of utility are lost while critical value thresholds are met. 

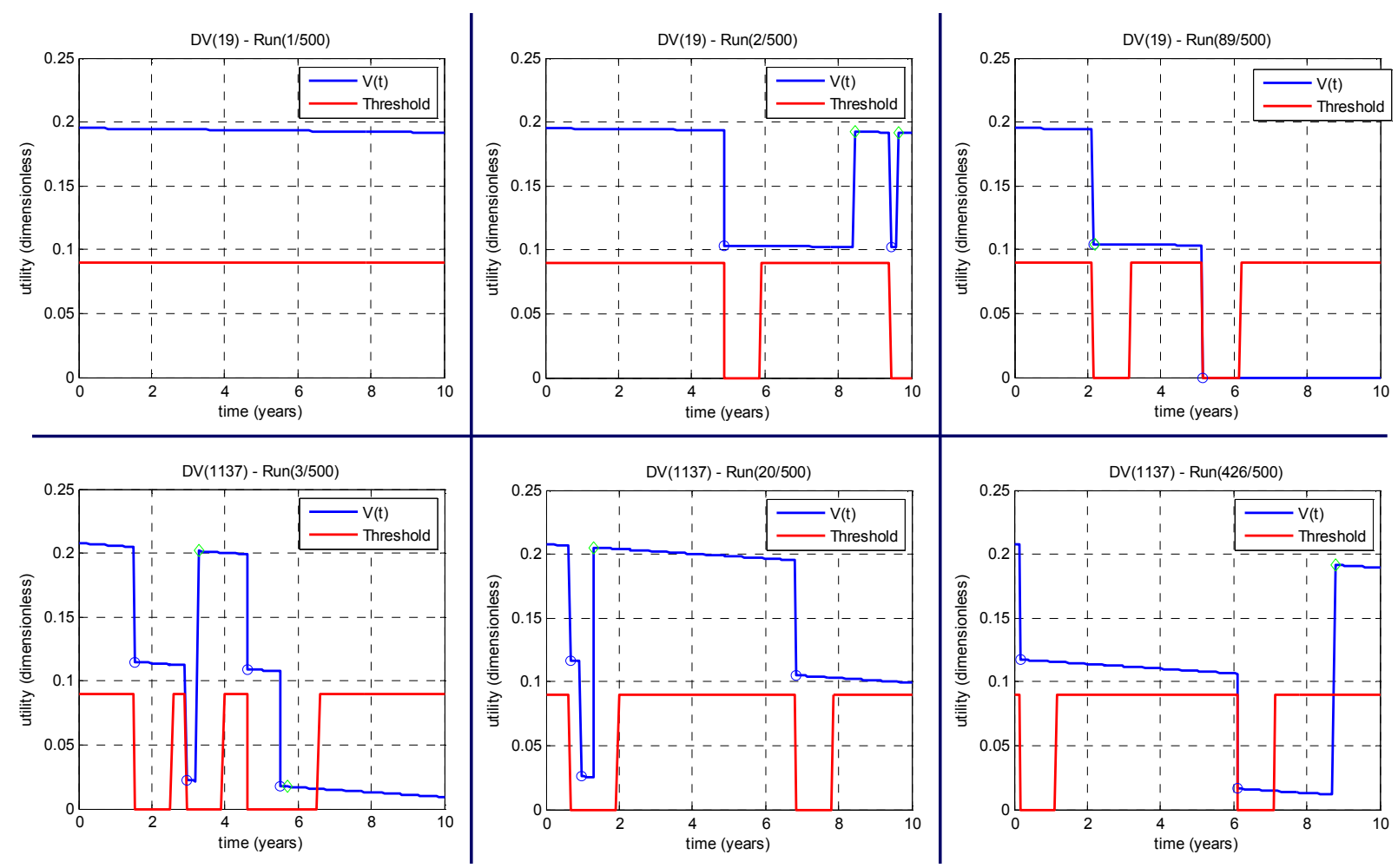

Figure 11. Sample Utility Trajectory Outputs from Design Vectors 19 and 1137

Figure 11 depicts six more sample utility trajectories. The top row illustrates the outcomes from three Monte Carlo runs of design vector 19, DV(19), and the lower row illustrates three runs of DV(1137). The cell in the upper left depicts the most common utility trajectory output from the model: slight utility degradation over time without any debris impacts. While the middle upper cell shows DV(19) receiving (and recovering from) two noncatastrophic debris impacts, the upper right cell shows the mission ending prematurely at year five from a catastrophic impact. Similar erratic utility trajectories are observed across the lower row. Interestingly, while both runs 3 (bottom left) and 426 (bottom right) of DV(1137) reveal an active space tug at end-of-life, both spend at least a year below $V_{x}$ due to failed and delayed servicing operations, respectively.

As survivability is a stochastic, path-dependent property, the outcome of any particular run for a given design vector is not necessarily representative or meaningful from a decision making perspective. Rather, each utility trajectory constitutes one data sample from a continuous distribution of potential system lifecycles. Furthermore, there is a need to distinguish across collections of utility trajectories of different design vectors (e.g., illustrated by rows in Figure 11). However, observing all 128,000 utility trajectories - 500 runs of each of the 2560 design vectors - is not practical from a decision making perspective. Therefore, the survivability metrics proposed in Section 3-time-weighted average utility/utility loss and threshold availability - are applied as aggregate measures for each set of space tug utility trajectories. 


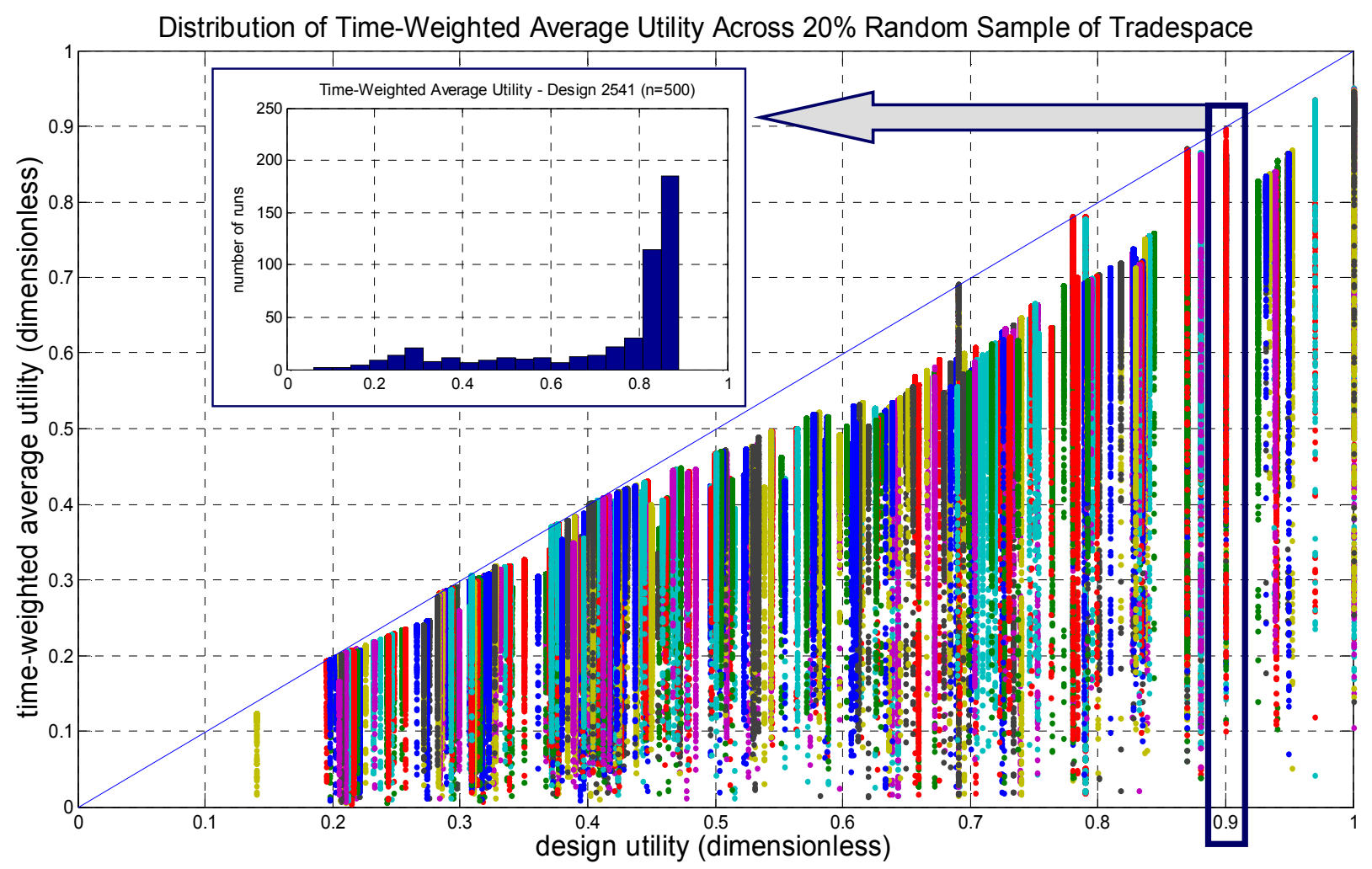

Figure 12. Distributions of Time-Weighted Average Utility

Figure 12 depicts the distributions of time-weighted average utility achieved by $20 \%$ of the design vectors over 500 Monte Carlo runs. (A random sample of $20 \%$ was selected to aid in information visualization.) Each column of identically-colored points represents the distribution of time-weighted average utility for a single space tug alternative. To organize the data, the columns are ordered along the horizontal axis in terms of design utility - the deterministic beginning-of-life utility $\left(V_{0}\right)$ achieved by a space tug before stochastic losses accrue from normal degradation and orbital debris. The design utility here is equivalent to the utility axis in the baseline tradespace (Figure 9). A $45^{\circ}$ line is also drawn to show the maximum time-weighted average utility value achievable.

The results are illuminating, showing a consistent pattern of highly-skewed distributions towards design utility. The histogram of DV(2541) in Figure 12 is displayed as a representative distribution of the highly-skewed behavior of time-weighted average utility across simulation runs. One interesting trend observed is the general regression of maximum time-weighed average utility values from $V_{0}$ for space tugs as design utility increases. This is due to both the increased susceptibility of larger (and generally higher-utility) space tug vehicles to orbital debris and to the lower $\Delta \mathrm{V}$ margins of the more massive space tug vehicles. (Given the full-factorial enumeration of design vectors, tradespace artifacts, such as the paring of low fuel mass with high-capability tugs, may result.) An additional behavior across the distributions is the presence of maximum time-weighted average utility outliers (in which no losses or degradation are observed vis-à-vis the design utility line) beginning around design utility values of 0.7 . These outliers are attributed to simulation runs of high-capability electric propulsion space tug designs that were set with excessive $\Delta \mathrm{V}$ margins and that did not suffer from orbital debris impacts.

The highly-skewed, long-tailed nature of the distributions of time-weighted average utility across simulation runs was also observed for the distributions of threshold availability. Figure 13 shows the output from $\mathrm{DV}(2541)$ utility trajectories as a sample distribution.

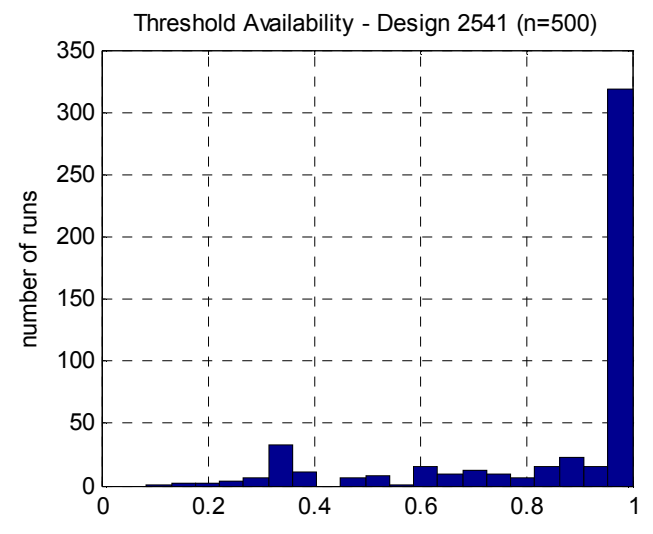

Figure 13. Sample Distribution of Threshold Availability 
Having generated a data set of utility trajectories and having applied two proposed measures of lifecycle survivability, we next compare the new metrics to existing survivability metrics and integrate the survivability metrics with measures of cost and design utility.
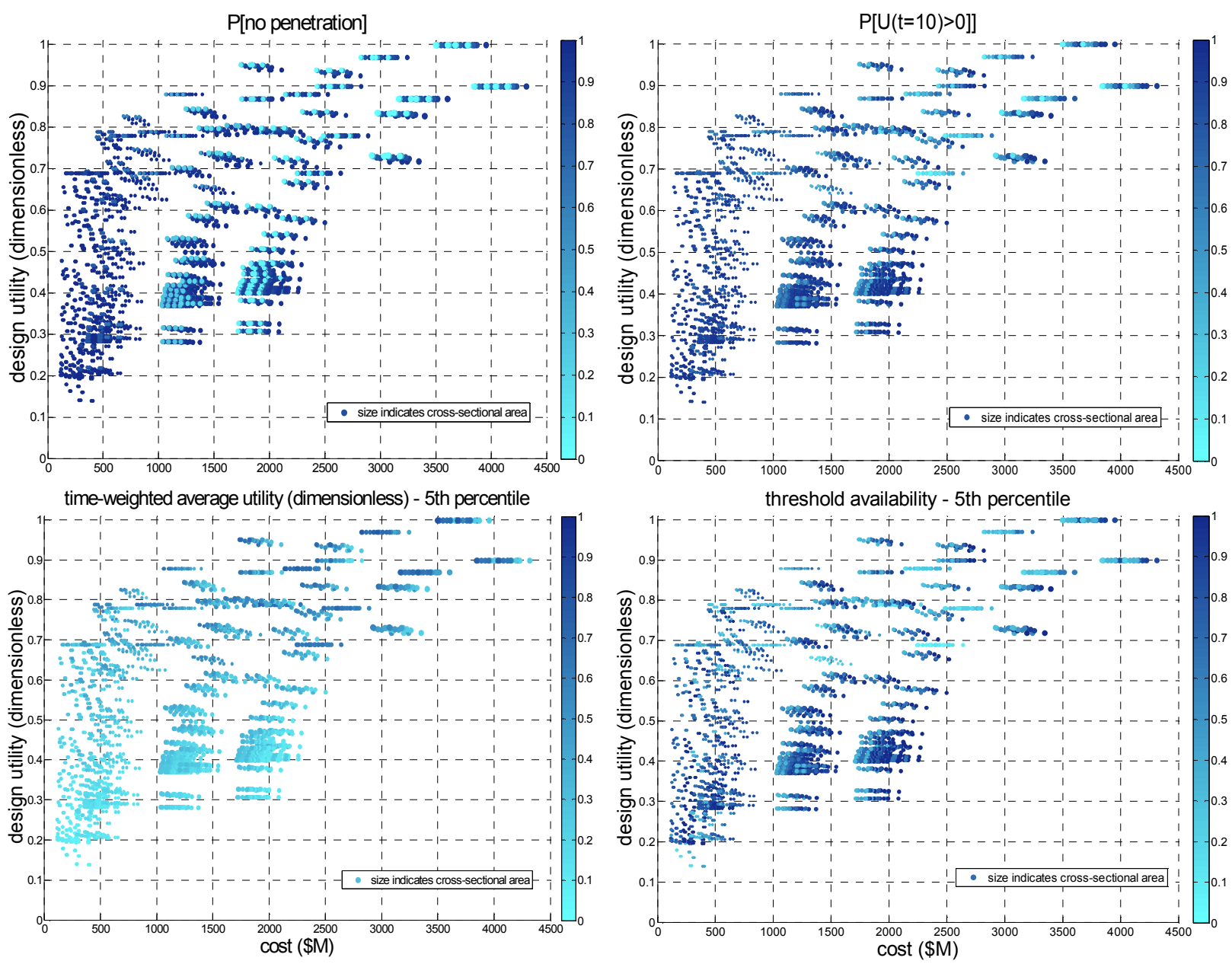

Figure 14. Application of Survivability Metrics to Baseline Tradespace

Figure 14 presents four separate survivability tradespaces, each incorporating one of four different survivability metrics to the baseline cost-utility tradespace in Figure 9. The top two tradespaces apply existing static conceptualizations of survivability while the bottom two tradespaces apply the proposed dynamic measures of survivability. Each survivability metric is depicted in color (i.e., darker means more survivable). In addition, to indicate the varying susceptibility of small and large space tugs to orbital debris, the size of each point in the tradespaces is correlated with exposed cross-sectional area.

The results across the four tradespaces vary tremendously, indicating the importance of understanding and selecting the correct metric or metrics for evaluating system survivability (i.e., depending on the metric chosen, very different conclusions may be drawn). The top-left tradespace, P[no penetration], shades each space tug design by the probability of no debris penetration over the 10-year operational life. In this representation, lower-cost designs achieve a high degree of survivability due to their low-susceptibility while the survivability of high-cost designs fluctuates as a function of shielding and collision avoidance. (Resilience enhancement strategies, such as servicing, are exogenous to this survivability metric.) The top-right tradespace, $\mathrm{P}[\mathrm{U}(\mathrm{t}=10)>0]$, is shaded by the probability of a non-zero utility (i.e., operational) space tug at end-of-life. Here, the high survivability of lower-cost designs is again observed. The survivability of high cost designs varies considerably as a function of all three survivability features.

The bottom-left tradespace in Figure 14, time-weighted average utility- $5^{\text {th }}$ percentile, shades each point by the level of time-weighted average utility achieved by $95 \%$ of the simulation runs of that design vector. For example, a 
value of 0.53 for time-weighted average utility $-5^{\text {th }}$ percentile means that $95 \%$ of the simulation runs of that design vector achieved a time-weighted average utility of at least 0.53 . (Rather than use a potentially misleading measure of central tendency such as average for the highly-skewed distributions, aggregate simulation results for timeweighted average utility and threshold availability are reported as percentiles.) The results here are naturally correlated with design utility. Many designs deemed survivable by this metric are found in the interior of the traditional cost-utility Pareto front. Interestingly, clusters of space tugs (varying only by survivability design variables) are observed where there is a non-monotonic relationship between adding levels of survivability features (indicated by rising costs) and time-weighted average utility. The bottom-right tradespace, threshold availability- $5^{\text {th }}$ percentile, shades each point by the proportion of time over the 10-year operational life spent above the critical value threshold. (As illustrated in Figure 11 and discussed in Section 2, this threshold varies depending on environmental context.) In contrast to time-weighted average utility, threshold availability generally rises as survivability features are added to each space tug. Threshold availability also appears to be sensitive to space tug size as smaller, less-susceptible designs achieve higher availability than their larger counterparts. Exceptions to these trends are also observed, including the low-availability of the extremely low-cost designs due to their tight performance margins.

The four tradespaces shown in Figure 14 present four conflicting (yet interdependent) perspectives regarding the survivability of the 2560 candidate space tug designs. Each tradespace presents useful information to the decision maker. However, there is a need to aggregate the information to allow integrated tradeoffs among other decision metrics for cost, performance, schedule, and risk. While the upper two tradespaces communicate the relative survivability of designs based on binary calculations of survivability, the two lower tradespaces are based upon the definition of survivability as a continuous, stochastic, and path-dependent property that is assessed over the entire operational life. Therefore, in order to incorporate all of the information contained in the utility trajectories (rather than small samples of the data such as the utility state at end-of-life), the next two tradespaces will focus on applying the new survivability metrics. 


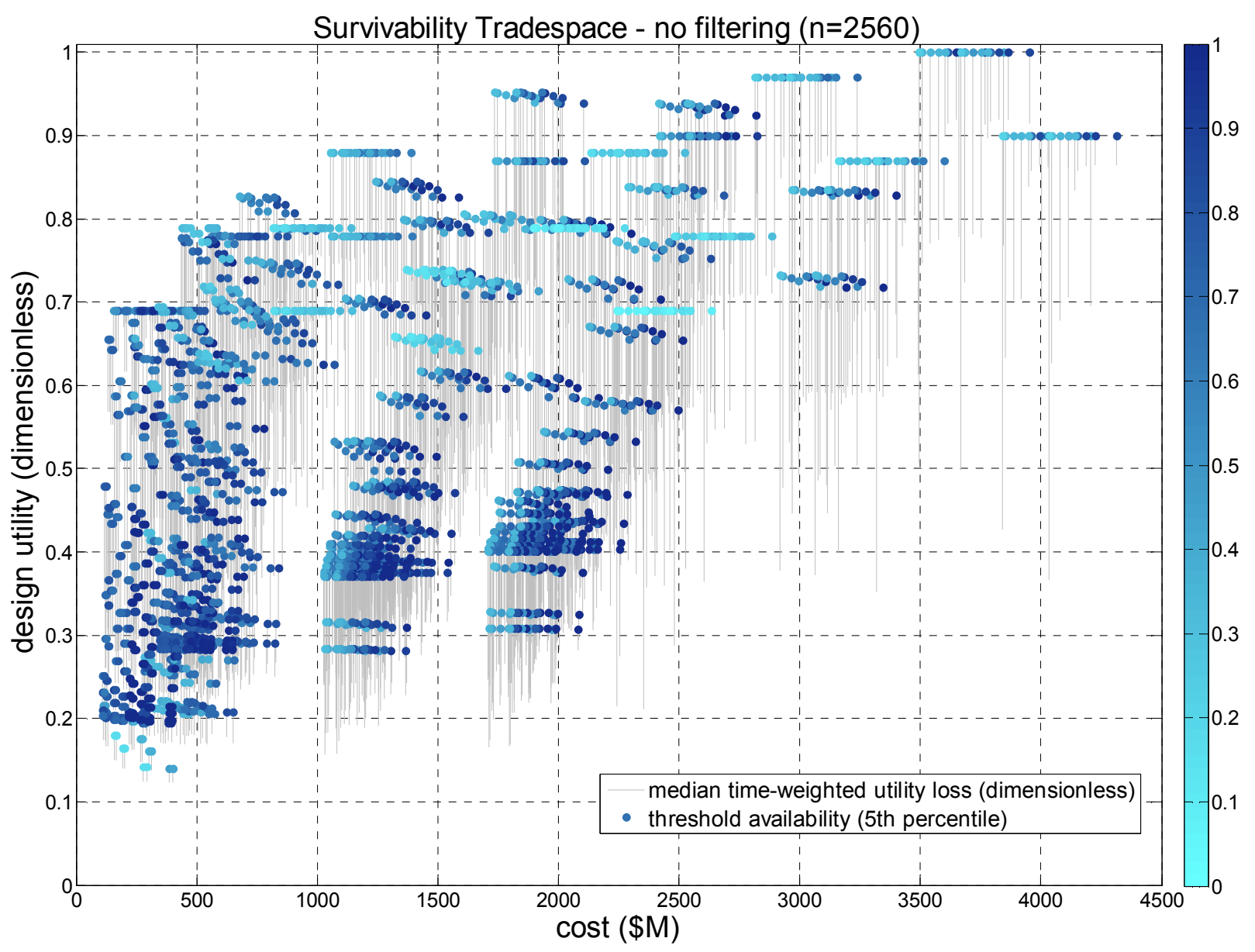

Figure 15. Survivability "Tear" Tradespace

To address the need for trading lifecycle cost, performance, and survivability of design alternatives, Figure 15 introduces a survivability "tear" tradespace representation. The purpose of the survivability "tear" tradespace is to integrate deterministic cost and design utility data with the distributions of time-weighted average utility and threshold availability. Preserving the cost and design utility axes of the baseline tradespace, the new survivability metrics are integrated using color (threshold availability $-5^{\text {th }}$ percentile) and a line drawn to the median timeweighted average utility. By definition (see Section 3B), the length of this line is the median time-weighted utility loss across the 500 utility trajectories for each space tug design. Rather than the $5^{\text {th }}$ percentile, the median is selected for summarizing the distribution of time-weighted utility loss for two reasons: (1) easing visualization for this particular data set by shortening the length of utility loss tails (from $5^{\text {th }}$ to $50^{\text {th }}$ percentile) and (2) recognition that decision makers are likely to be more risk-averse regarding threshold availability (a construct for measuring assured access to some level of minimum capability) than time-weighted average utility (a construct for assessing degree of degradation).

Some interesting trends can be observed in Figure 15, such as the poor performance of very low-cost and very expensive designs in terms of threshold availability (due to low-performance margins and high-susceptibility, respectively). Irregular reductions in utility loss can be observed as survivability features are added to clusters of baseline space tug designs. Smaller utility losses appear to occur in the lower-cost (smaller), lower-susceptibility region of the tradespace. Unfortunately, one consequence of tagging each of the 2560 design alternatives with four data attributes is that Figure 15 becomes very concentrated and difficult to interpret. 


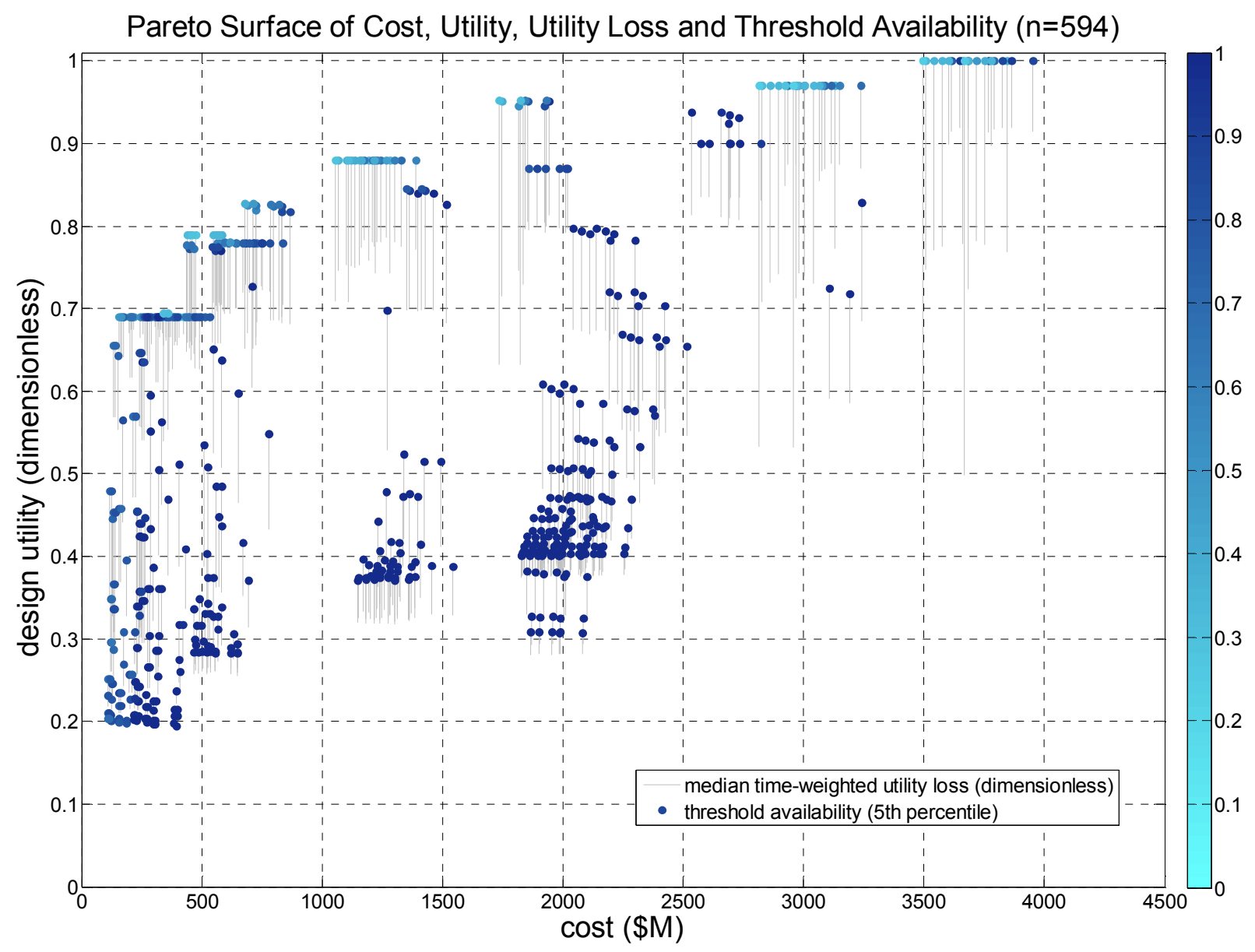

Figure 16. Pareto Surface of Survivability Tradespace

Figure 16 reduces the concentration of Figure 15 by filtering the 2560 designs for Pareto-optimality over the four decision metrics: cost, design utility, median time-weighted utility loss, and threshold availability. When considering the tradespace in four dimensions, the surface of Pareto efficient designs contains three individual Pareto sets (as determined by a projection onto the cost-utility, cost-availability, cost-utility loss planes) as well as other points that would not project onto any of these two-dimensional surfaces. These other points exist at the tradeoffs among design utility, threshold availability, and time-weighted utility loss for the decision maker and represent "compromise" value solutions. ${ }^{\dagger \dagger}$

One implication of filtering over the four decision metrics is that several interior designs become Pareto-efficient based upon their superior performance in terms of survivability. Rather than only allowing the selection of designs dominant in terms of cost and utility at beginning-of-life, the filtered survivability "tear" tradespace presents alternative designs that may offer superior performance over a lifecycle of disturbances. The results in Figure 16 illustrate the potential value of this method: most of the designs in the cost-utility Pareto front have much lower threshold availability - and considerably higher utility loss - than interior designs while several of the highlysurvivable space tugs are only slightly dominated in terms of cost and design utility.

Having proposed a survivability "tear" tradespace for enabling integrated trades to be made across alternative concepts in terms of cost, performance, and survivability, the impact of each of the three survivability design

\footnotetext{
${ }^{\dagger \dagger}$ Counterintuitively, as decision metric axes are added to filter for Pareto-efficiency, more designs will remain after filtering in the survivability "tear" tradespace. This is because as each axis is added, a new two-dimensional Pareto front is created with a new set of projected compromise solutions. For example, a cost-utility filter applied to Figure 15 leads to 111 Pareto-efficient designs while a cost-utility-utility loss filter leads to 279 Pareto-efficient designs. As a fourth metric is added for filtering (as in Figure 16), 594 designs are deemed Pareto-efficient. This growth of Pareto-efficient designs as decision metrics are added is analogous to the multi-stakeholder problem in utility theory. ${ }^{19}$
} 
variables on the survivability of each of the 128 baseline designs (i.e., full-factorial of first three, non-survivability design variables in Figure 5) is now examined. This analysis may be useful for maximizing the survivability of an individual space tug (if a baseline design concept has already been selected) as well as for investigating the overall sensitivity of space tug survivability to shielding, active collision avoidance, and on-orbit servicing.

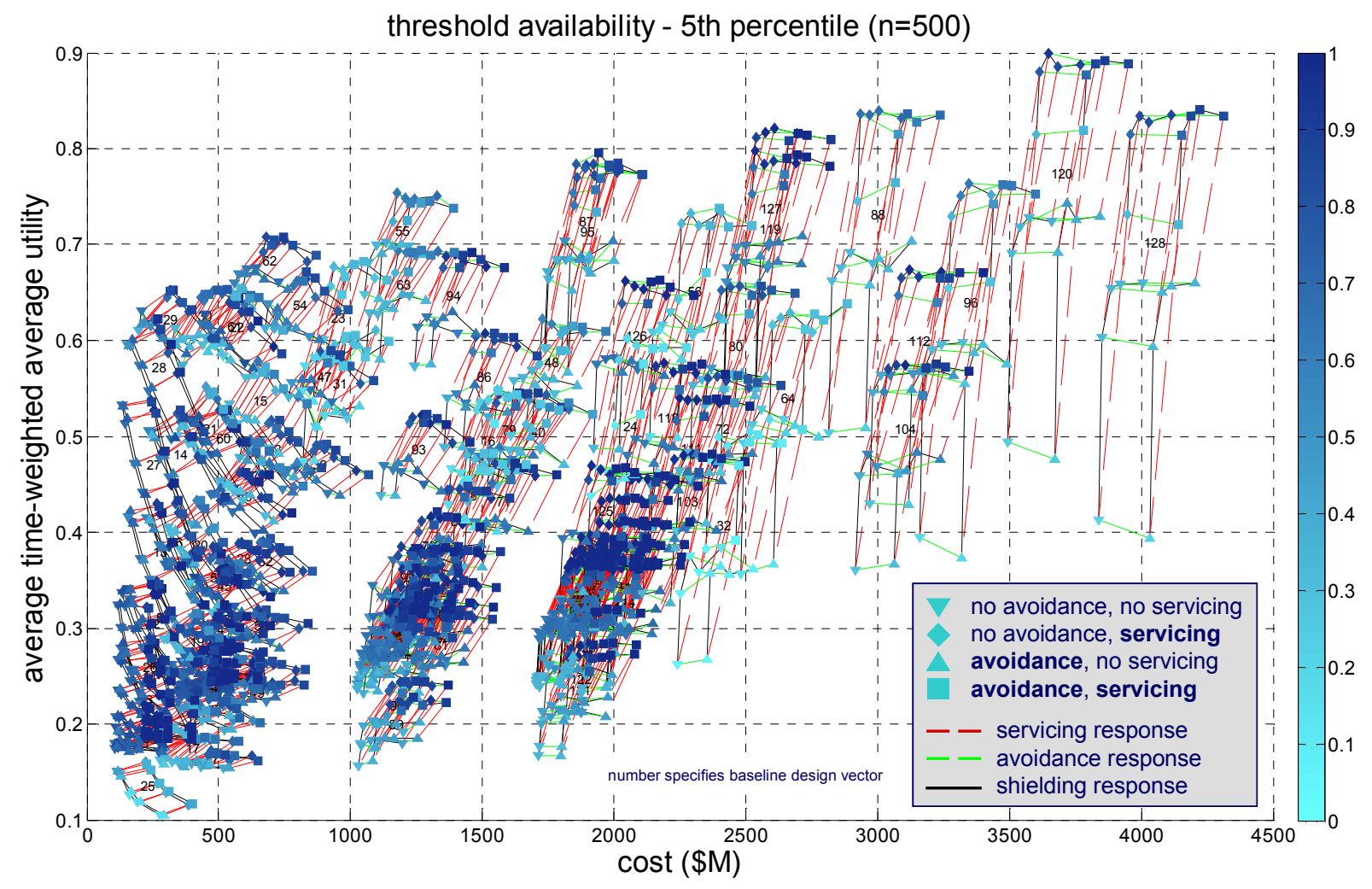

Figure 17. Survivability Response Surfaces - 128 Baseline Designs

Figure 17 plots survivability response surfaces for each of the baseline designs. Each design point is located in terms of cost and average time-weighted average utility ${ }^{\sharp t}$ and shaded by $5^{\text {th }}$ percentile threshold availability. Linked clusters (labeled 1 through 128) indicate a common baseline space tug of fixed manipulator mass, propulsion type, and fuel load. The impact of survivability features is evaluated by finding the lowest-cost inverted triangle in each cluster to identify the baseline space tug design (which incorporates only $30 \mathrm{~kg}$ of shielding and no avoidance or servicing). Then, the response surfaces for shielding, servicing, and avoidance may be identified by examining the solid black, dashed red, and dashed green lines, respectively. Designs incorporating no servicing are represented with three-sided shapes (i.e., as triangles for designs with avoidance and as inverted triangles for designs with no avoidance) while designs that do incorporate servicing are represented as four-sided shapes (i.e., as squares for designs with avoidance and as diamonds for designs with no avoidance).

Interesting trends may be indentified in the (relatively) sparse, high-cost region of Figure 17. For example, increasing the shielding appears to increase threshold availability as shielding mass is increased, while average utility benefits only from the initial increases in shield mass. Avoidance appears to have no statistically significant impact across the 500 runs (as availability is constant and average utility fluctuates slightly up or down). Servicing has a very positive impact on both survivability metrics in this region of the tradespace. Unfortunately, it is hard to extract survivability response surface trends across this display of the entire tradespace given the density of clusters in lower-cost regions.

\footnotetext{
$\$$ Time-weighted average utility is the average across a single trajectory while average time-weighted average utility is the average of this value across the set of 500 Monte Carlo trials.
} 


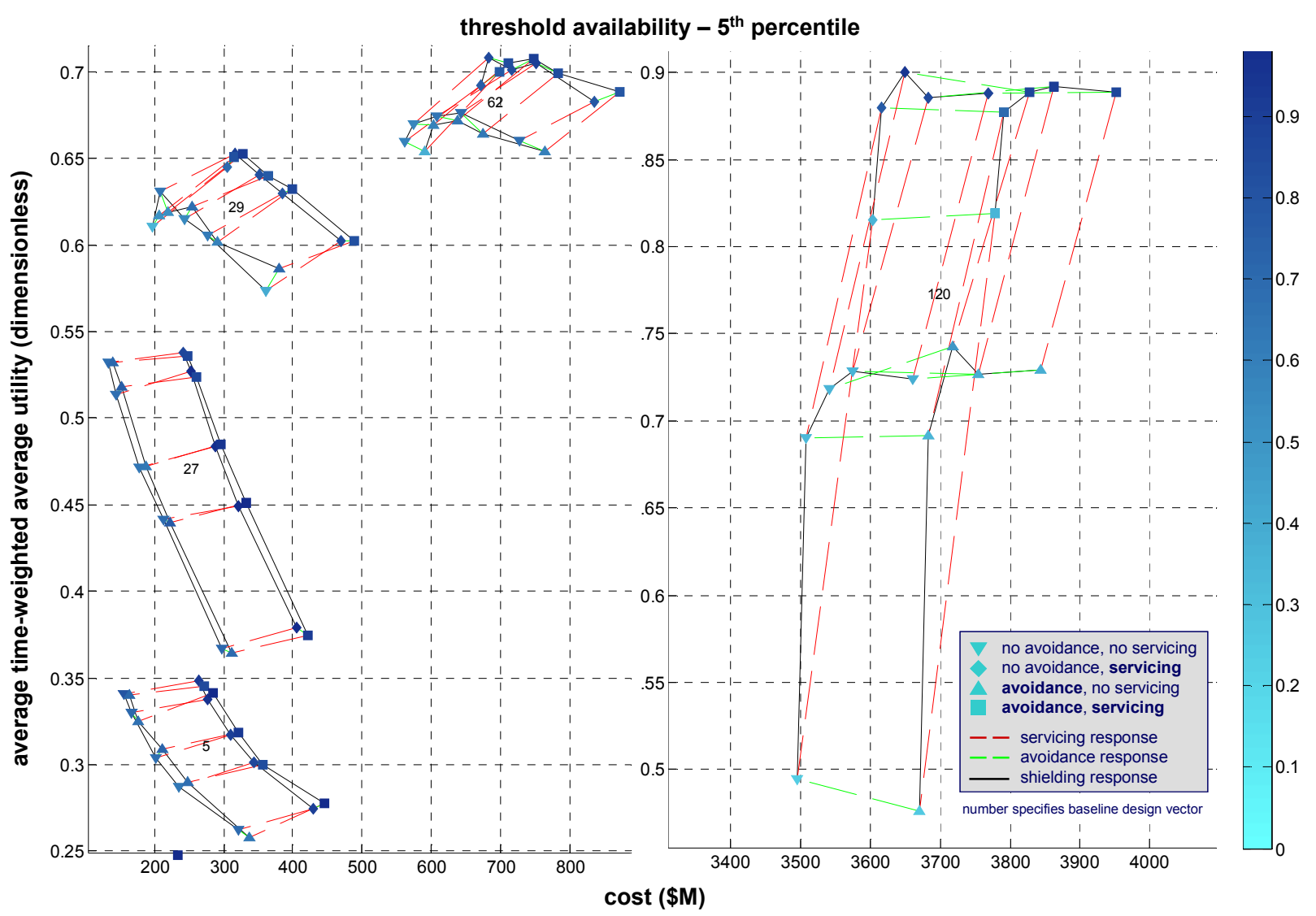

Figure 18. Variation in Survivability Response Surfaces

To compare survivability response surfaces in different regions of the tradespace, Figure 18 magnifies five lowcost designs and one high-cost design from Figure 17. While the width and height of each magnification is conserved to enable comparison, the cost and average utility axes are necessarily different. At this higher resolution, the impact of shielding, avoidance, and servicing on both individual tug designs and different regions of the tradespace can be better observed.

The impact of shielding on average utility and threshold availability is very different across these regions of the tradespace. For baseline design number 5, $\mathrm{BD}(5)$, shielding has a negative impact on both cost and average utility while providing no appreciable gains in threshold availability. This is also true of $\mathrm{BD}(27)$. The shielding impact on $\mathrm{BD}(5)$ and $\mathrm{BD}(27)$ stands in stark contrast to $\mathrm{BD}(120)$ where the first two increases in shielding mass result in large increases in average utility and threshold availability at only marginal increases in cost. However, after the first two increases in shielding mass in $\mathrm{BD}(120)$, survivability benefits diminish while dry mass penalties accumulate. $\mathrm{BD}(29)$ and $\mathrm{BD}(62)$ represent intermediate cases where the first increase in shielding mass (from 30 to $100 \mathrm{~kg}$ ) yields slight improvements in average utility and threshold availability before negative returns occur at $300 \mathrm{~kg}$ of shielding.

Avoidance does not appear to have a significant impact on survivability in either region of the tradespace. The only general trend observed is the assumed 5\% cost penalty associated with incorporating active collision avoidance. Indeed, some designs even occasionally show losses in average utility for incorporating active collision avoidance (despite the fact that, as modeled, avoidance can only improve utility trajectories). The limited impact of avoidance is due to the fact that detectable debris encounters (assumed to be $>10 \mathrm{~cm}$ in diameter) are extremely rare events. Therefore, the avoidance response surface is horizontal to account for the cost penalty while slight, vertical fluctuations may be attributed to random variation over the 500 Monte Carlo trials.

Unlike shielding and avoidance, servicing has a positive impact on average utility and threshold availability across the tradespace. The impact is not uniform, however, as $\mathrm{BD}(120)$ (with its higher exposed cross-sectional area) is more susceptible to debris impacts. This space tug is much more likely to experience multiple, noncatastrophic debris impacts over its operational life and therefore benefit substantially from servicing (increasing 
average utility approximately $25 \%$ ). Servicing has a significant yet smaller positive impact on the lower-end designs, particularly in terms of threshold availability.

\section{Conclusions}

\section{A. Discussion}

The process of developing survivability metrics and applying them within the dynamic survivability model yielded several insights for the space tug tradespace as well as implications for the proposed metrics and ideas for future work. In terms of space tug survivability, one interesting result is the criticality of the inherent survivability derived from the baseline design vector (before incorporating survivability design variables). For example, selecting baseline tugs with smaller cross-sectional areas has a much greater impact on susceptibility reduction than active collision avoidance. Similarly, increasing grappling capability margin has a greater impact on reducing vulnerability than bumper shielding. Another modeling insight is the extreme sensitivity of the results to the damage model for small- and medium-sized impacts (for which limited empirical data exist). Of all the results, Figure 16 is the most interesting from a decision-making perspective as it reveals that the cost-utility Pareto-optimal designs exhibit poor survivability while many highly survivable designs are only slightly dominated in terms of cost and utility.

The survivability metrics of time-weighted average utility/utility loss and threshold availability were applied successfully to the space tug data set. The metrics are unique but interdependent. For example, high average utility implies high threshold availability (although high threshold availability does not imply high average utility). Taken together, the metrics served as powerful discriminators of survivability performance.

Both proposed metrics are closely related to existing metrics in the literature. Threshold availability is essentially a modification of inherent availability that allows for a variable threshold of stakeholder expectations. Time-weighted average utility is analogous to the Quality-Adjusted Life Year (QALY). Often used in the medical community for patients evaluating treating options, QALY is a multi-attribute quantity that varies as a function of time. QALYs scale the length of each year of remaining life by the quality of life expected in that year. The scaled years are then added to form QALYs. Thus, many years of low quality are equivalent to fewer years of high quality. ${ }^{30}$

\section{B. Future Work}

Looking ahead, there are multiple options to improve the space tug model and advance the survivability tradespace methodology. First, many tradespace artifacts might be removed (e.g., pairing of extremely high-levels of shielding with small tugs) if the strict full-factorial enumeration of the design space (Figure 5) is replaced with a non-absolute design vector construction. For example, fuel mass might be reduced in the case of electric propulsion. Additionally, the shield mass design variable might be replaced with shield thickness. Second, more fidelity might be added to the satellite damage and servicing models.

Third, future models should strive to incorporate all path dependencies in disturbance origination and utility trajectories. As currently modeled, only path-dependent vulnerability is modeled by tracking the cumulative effect of non-catastrophic debris impacts in the utility trajectories. However susceptibility and resilience are also pathdependent properties. For example, satellite susceptibility to debris may be elevated in the time period immediately following a local breakup. Resilience path-dependencies are not included in the current model which assumes that the servicer is always available when needed. Given the small cost-increment assigned to servicing, it is more likely that servicing will be a shared resource financed through insurance payments from many spacecraft. The system-ofsystems formed by these spacecraft and the servicing infrastructure will collectively impact the survivability of each individual spacecraft. In such a shared servicing architecture, the usefulness of servicing will therefore depend upon the design choices of the other customers utilizing the servicing infrastructure. For example, servicing responsiveness could decrease as other spacecraft make design choices that lead to greater susceptibility and therefore use the servicing infrastructure more often. Ensuring fair access to a jointly-financed infrastructure is a key policy problem for designing survivable systems that depend on a shared resource to reduce vulnerability or enhance resilience.

Fourth, a factor analysis of the survivability metrics might be performed to better understand the interdependencies among the metrics. Fifth, while the space tug example indicates that the proposed metrics can be applied prescriptively to the evaluation of the survivability of alternative satellite designs, general lessons from the modeling effort should be extracted to develop an integrated design methodology for survivability. 


\section{Acknowledgments}

Funding for this work was provided by the Systems Engineering Advancement Research Initiative (seari.mit.edu), a consortium of systems engineering leaders from industry, government, and academia; and the Program on Emerging Technologies (PoET), an interdisciplinary research effort of the National Science Foundation at MIT.

\section{References}

${ }^{1}$ Richards, M., D. Hastings, D. Rhodes, and A. Weigel, "Defining Survivability for Engineering Systems," $5^{\text {th }}$ Conference on Systems Engineering Research, Hoboken, NJ., 2007.

${ }^{2}$ GAO, "Critical Infrastructure: Commercial Satellite Security Should Be More Fully Addressed," Report to U.S. Senate, 2002.

${ }^{3}$ Ballhaus, W., "Successes and Challenges in Transforming National Security Space," 43rd Aerospace Sciences Meeting, Reno, NV, 2005.

${ }^{4}$ Rumsfeld, D., D. Andrews, R. Davis, H. Estes, R. Fogleman, J. Garner, W. Graham, C. Horner, D. Jeremiah, T. Moorman, D. Necessary, G. Otis and M. Wallop, "Report of the Commission to Assess United States National Security Space Management and Organization," 2001.

${ }^{5}$ Wilson, T., "Threats to United States Space Capabilities," Prepared for the Commission to Assess United States National Security Space Management and Organization, January 2001

${ }^{6}$ Covault, C., "Space Control: Chinese anti-satellite weapon test will intensify funding and global policy debate on the military uses of space," Aviation Week and Space Technology, 22 Jan. 2007, pp. 24-25.

${ }^{7}$ Mowthorpe, M., "US Military Space Policy 1945-92." Space Policy, Vol. 18, No. 1, 2002, pp. 25-36.

8 "U.S. National Space Policy," White House Office of Science and Technology Policy, 31 August 2006.

${ }^{9}$ Ball, R. and M. Kolleck, "Survivability: It's Not Just for Aircraft Anymore," Aircraft Survivability, Winter 2000, pp. 10-11.

${ }^{10}$ Long, A., M. Richards and D. Hastings, "On-Orbit Servicing: A New Value Proposition for Satellite Design and Operation," Journal of Spacecraft and Rockets, Vol. 44, No. 4, 2007, pp. 964-976.

${ }^{11}$ Brown, O., "Speech by Dr. Owen Brown on Fractionated Spacecraft," Anaheim, CA, 2007.

12 Richards, M., D. Hastings, D. Rhodes and A. Weigel, "Systems Architecting for Survivability: Limitations of Existing Methods for Aerospace Systems," 6 ${ }^{\text {th }}$ Conference on Systems Engineering Research, Los Angeles, CA, 2008.

${ }^{13}$ McManus, H., Richards, M., Ross, A., and Hastings, D., "A Framework for Incorporating "ilities" in Tradespace Studies," AIAA Space 2007, Long Beach, CA, 2007.

${ }^{14}$ Wertz, J., and W. Larson, Space Mission Analysis and Design, Microcosm Press, El Segundo, 1999.

${ }^{15}$ Klinkrad, H, Space Debris Models and Risk Analysis, Springer, Chichester, 2006

${ }^{16}$ Ross, A., D. Hastings, J. Warmkessel and N. Diller, "Multi-Attribute Tradespace Exploration as Front End for Effective Space System Design," Journal of Spacecraft and Rockets, Vol. 41, No. 1, 2004, pp. 20-28.

${ }^{17}$ Ross, A., and Rhodes, D., "Using Natural Value-Centric Time Scales for Conceptualizing System Timelines Through Epoch-Era Analysis," $18^{\text {th }}$ INCOSE Symposium, Utrecht, Netherlands, 2008.

${ }^{18}$ McManus, H., and Schuman, T., "Understanding the Orbital Transfer Vehicle Tradespace," AIAA Space 2003, Long Beach, CA, 2003.

${ }^{19}$ Keeney, R. and H. Raiffa, Decisions with Multiple Objectives: Preferences and Value Tradeoffs, Cambridge University Press, Cambridge, 1993.

20 "SMC Systems Engineering Primer and Handbook," Space \& Missile Systems Center, U.S. Air Force, 2005.

${ }^{21}$ Keeney, R., Value-Focused Thinking: A Path to Creative Decisionmaking, Harvard University Press, Cambridge, 1992.

${ }^{22}$ Shaw, G., D. Miller and D. Hastings, "Development of the Quantitative Generalized Information Network Analysis Methodology for Satellite Systems," Journal of Spacecraft and Rockets, Vol. 38, No. 2, 2001, pp. 257-269.

${ }^{23}$ Bayer, T., "Planning for the Un-plannable: Redundancy, Fault Protection, Contingency Planning and Anomaly Response for the Mars Reconnaissance Orbiter Mission," AIAA Space 2007, Long Beach, CA, 2007.

${ }^{24}$ Ball, R., The Fundamentals of Aircraft Combat Survivability Analysis and Design, AIAA, Reston, 2003.

${ }^{25}$ Blanchard, B. and W. Fabrycky, Systems Engineering and Analysis, Prentice Hall, Upper Saddle River, 2006.

${ }^{26}$ Richards, M., Springmann, P., McVey, M., "Assessing the Challenges to a Geosynchronous Space Tug Vehicle," Proceedings of SPIE Defense and Security Symposium, Vol. 5799, The International Society for Optical Engineering, Bellingham, WA, 2005.

${ }^{27}$ McVey, M., "Valuation Techniques for Complex Space Systems: An Analysis of a Potential Satellite Servicing Market," Master's thesis, Department of Aeronautics and Astronautics, Massachusetts Institute of Technology, Cambridge, MA, 2002.

${ }^{28}$ Remo, J., "Orbital Debris Effects from Space-Based Ballistic Missile Interception," Journal of Spacecraft and Rockets, Vol. 42, No. 3, 2005, pp. 487-492.

${ }^{29}$ Badhwar, G. and P. Anz-Meador, "Determination of the Area and Mass Distribution of Orbital Debris Fragments," Earth, Moon, and Planets, Vol. 45, 1989, pp. 29-51.

${ }^{30}$ Johannesson, M., "The Ranking Properties of Healthy-Years Equivalents and Quality Adjusted Life-Years Under Certainty and Uncertainty," International Journal of Technology Assessment in Health Care, Vol. 11, No. 1, 1995, pp. 40-48. 